\title{
核磁共振残留偶极耦合参数在有机分子结构 鉴定中的应用
}

\author{
李高伟 ${ }^{2}$, 雷新响 ${ }^{*}$ \\ 1. 中南民族大学药学院, 武汉 430074 \\ 2. 商丘师范学院化学化工学院, 商丘 476000 \\ *通讯作者, E-mail: xxlei@mail.scuec.edu.cn
}

收稿日期: 2017-07-17; 接受日期: 2017-09-15; 网络版发表日期: 2017-11-24

国家自然科学基金(编号：21572164)和中德中心科学基金(编号：GZ1289)资助项目

\begin{abstract}
摘要残留偶极耦合(residual dipolar coupling, RDC)作为核磁共振各向异性参数在解析有机分子构型及优 势构象等方面的应用具有强有力的优势, 它能反映分子中原子在磁场中的空间距离与角度信息, 实现分子三 维空间的构建. 本文对残留偶极耦合在有机分子结构鉴定方面的进展进行综述, 详细总结了测定RDC的定 向介质和检测分析方法, 并利用实例展示RDC在天然产物、合成药物、有机反应中间体或络合物, 以及对映 异构体等立体化学方面的分析与应用.
\end{abstract}

关键词核磁共振波谱,残留偶极耦合, 定向介质,有机小分子,结构分析

\section{1 引言}

天然或合成有机分子的结构鉴定, 特别是立体化 学解析一直是有机化学和药物化学的核心任务之一, 而核磁共振波谱在其结构鉴定与分析方面具有不可替 代的作用. 作为经典的核磁共振参数: 化学位移 $(\delta)$, 耦 合常数 $(J)$ 以及奥弗豪塞尔核 (nuclear overhauser effect, $\mathrm{NOE}$ ) 效应, 是有机化学家日常实验中用于鉴定有机小 分子和生物大分子结构的常用信息. 由于化学位移 $\delta$ 受到周围化学环境的影响很大, 所以仅能有一个定性 作用; 无论同核还是异核 $J$ 耦合, ${ }^{3} J$ 耦合常数是结构计 算的重要约束条件, 它通过Karplus经验公式与二面角 联系起来, 然而 ${ }^{3}$ 耦合仅局限于不超过 3 个共价键所形 成的二面角; 而NOE效应是空间距离较近的两个(组)
质子由于相互弛豫而形成的相互映照, 可以把相隔多 个化学键的两个质子在一定的空间距离内关联起来, 对于 ${ }^{1} \mathrm{H}$ 核来说, 当距离大于 $5 \AA$ 时, NOE作用有限. 因 此, 基于 $\mathrm{NOE}$ 和 ${ }^{3}$ 耦合常数提供的仅是局部结构信息, 对于分子中相距较远片段或被惰性核阻断后的相对 构型分析则比较困难.

偶极耦合是两个磁性核通过空间传递的相互作 用, 在固体核磁共振中, 偶极耦合的数值通常在数千 赫兹, 偶极耦合作用太强使谱峰变得极宽, 分辨率差 而无法解析; 而在溶液中, 由于分子的布朗运动, 偶极 耦合作用又被平均为零, 尽管此时测得的谱图分辨率 高却失掉了附加在偶极耦合中的结构信息. 然而, 当 溶液中分子的运动受到限制的时候, 偶极耦合可能没 有被完全平均掉, 而保留较小的残留值, 称为残留偶

引用格式： 李高伟,雷新响. 核磁共振残留偶极耦合参数在有机分子结构鉴定中的应用. 中国科学: 化学, 2017, 47: 1401-1423

Li G, Lei X. Structural elucidation of organic molecule from residual dipolar couplings. Sci Sin Chim, 2017, 47: 1401-1423, doi: 10.1360/N03201700124 
极耦合 (residual dipolar coupling, RDC) ${ }^{[1 \sim 12]}$. RDC可以 提供两个直接相连或者非直接相连的核之间的结构 信息, 其大小与两个核之间的距离以及两个核之间的 键矢量相对于外加磁场的角度取向相关, 其值通常用 $D_{i s}$ 表示, 其中 $\mu_{0}$ 是空间的介电常数, $h$ 为普朗克常数, $\gamma$ 为磁旋比, $r_{i s}$ 表示两个自旋核之间的矢量距离, $\theta$ 表示 $i$ 和 $s$ 的核间矢量相对外加磁场之间的角度大小(图1). 因此, RDC值与原子的空间排列相关, 能反映分子的 构型以及优势构象, 是研究分子立体结构的一个重要 核磁共振参数, 与NOE和 ${ }^{3} J$ 耦合常数的信息互补 ${ }^{[13,14]}$.

近年来, 随着该领域研究的深入, 残留偶极耦合 参数正逐渐在有机分子三维结构(构型和构象)测定中 发挥重要作用, 受到广泛关注 ${ }^{[15-20]}$. 本文综述了近十 几年来发展的有机小分子残留偶极耦合测定的定向 介质及脉冲方法, 并总结利用该各向异性参数来解决 天然产物立体结构、有机反应中间体测定及手性对 映异构体区分等实例.

\section{2 定向介质}

准确测定RDC必须满足两个条件:一是通过一定 的方法使分子的运动受限或约束其变为各向异性, 使 偶极耦合作用不能完全抵消, 从而产生较弱的“残留 偶极耦合”; 二是偶极耦合作用不能太强, 使得图谱质 量要高分辨, 线宽窄以便于提取 RDC值. 因此, 必须将 溶质分子置于一个非各向同性环境, 即一种定向介质 (alignment media)中, 这是测定有机分子 $\mathrm{RDC}$ 的前提. 目前, 发展和构建结构新颖、简便实用并与有机溶剂 兼容的 RDC提取介质及方法成为有机化学工作者迫 切追求的目标.

1963年, Saupe教授等 ${ }^{[21,22]}$ 报道了第一张液晶相中 有机小分子氢谱: 将苯溶于氧化偶氮苯甲醚液晶介 质中得到 $\mathrm{H}-\mathrm{H}$ 耦合高达 $2.5 \mathrm{kHz}$ 的多重态谱, 遗憾的是,
耦合太强而无法实际应用. 40年后, 随着 RDC理论的 日臻完善, 越来越多的定向介质被开发并加以实际应 用 ${ }^{[23-26]}$. 目前用于测定有机分子的RDC主要有两类定 向介质：能溶胀且可拉伸或挤压的凝胶 $(\mathrm{SAG})$, 以及溶 致液晶(LLC). 凝胶介质的定向原理是先通过溶剂溶 胀(一般时间较长), 将待测分子机械地限定或约束于 凝胶空腔内, 使其各向同性的布朗运动受限; 而具有 一定排列取向的溶致液晶可使其取向性自发地部分 转移到被分析物, 从而具有约束. 这些定向介质都可 以在磁场中产生弱的定向排列, 待测有机小分子通过 与定向介质之间的相互作用会产生适度的定向性质, 从而测定其残留的偶极-偶极相互作用 (即RDC, $D_{i s}$ ).

\section{1 可拉伸或压缩凝胶}

凝胶是胶体粒子或高分子在一定条件下互相连 接形成的空间网状结构, 结构空隙中可填充分散介质 的一种特殊的分散体系. 为了能准确测量RDC, 同时 获得分辨率尽可能高的谱图, 需要对定向凝胶的强度 进行调节. 交联凝胶的定向强度与胶的本身性质(交 联度)和棒状凝胶的直径有关. 定向角度可以通过准 备多个不同规格的凝胶样品, 分别在核磁管内沿管壁 轴向与横向自由溶胀后探究最优的定向条件 ${ }^{[27]}$; 也可 以借助最早由德国Luy教授 ${ }^{[28,29]}$ 发明的拉伸装置工具, 通过改变单个样品的排列强度找出适度的定向条件 (图2). 根据凝胶在有机溶剂中的溶解极性, 将凝胶类 定向介质分为以下 3 类定向胶.

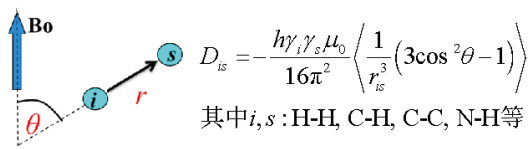

图 1 两磁性核间的残留偶极耦合值计算原理及公式 ${ }^{[13,14]}($ 网 络版彩图)

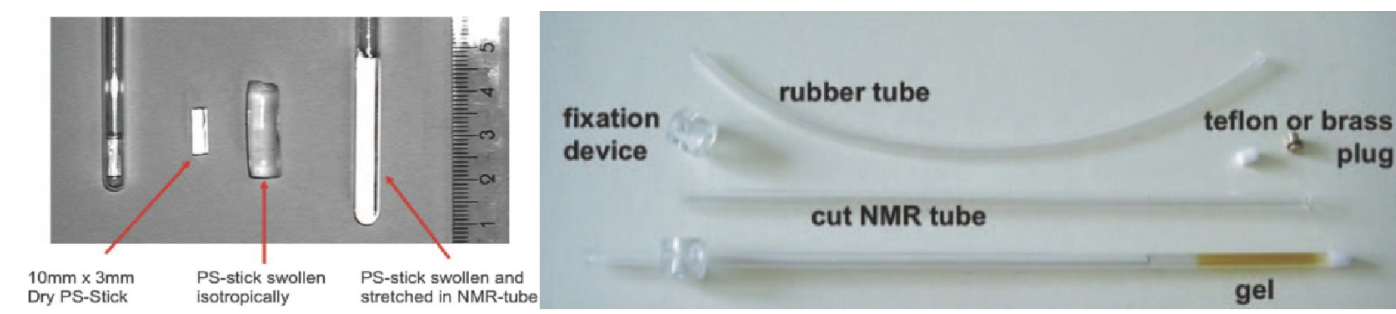

图 2 核磁管内自由溶胀的凝胶 ${ }^{[27]}$ 和拉伸凝胶核磁装置 ${ }^{[28,29]}$ (网络版彩图) 


\subsection{1 非极性有机溶剂定向胶}

交联聚苯乙烯胶(PS) 是利用过氧二酰苯(DBP)引 发二乙烯基苯(DVB)与苯乙烯发生聚合反应, 得到可 溶于常规非极性有机溶剂的胶, 其定向排列能力通过 改变单体的比例进行很好的控制 ${ }^{[27]}$. 由于PS胶的残留 背景峰太多, 增加了 $\mathrm{RDC}$ 的测量难度以及图谱解析困 难. 为了解决背景峰干扰的问题, 随后Luy等 ${ }^{\left[{ }^{[0]}\right.}$ 又发展 了全氛代聚苯乙烯凝胶( dPS胶), 大大降低了背景峰.

而利用 $10 \mathrm{MeV}$ 高压 $\beta$ 射线激发得到不同聚合链长 度及交联度的聚二甲基硅氧烷(PDMS)胶, 在各种非极 性有机溶剂中则表现出不同的定向能力. 通过对螺二 狮类化合物两种相对构型的RDC分析与结构验证, 从 而确立了PDMS的实际应用价值. 然而交联过程需用 到非常规的 $\beta$ 射线仪, 使PDMS胶不能商品化 ${ }^{[31]} .2017$ 年, Thiele等改用化学合成的方法制备PDMS胶, 以八甲 基环四硅氧烷 $\left(\mathrm{D}_{4}\right)$ 为原料先分别合成交联剂( $\left(\right.$ bis- $\left.\mathrm{D}_{4}\right)$ 和 引发剂, 然后三组分再聚合产生非活性的PDMS, 最后 $150^{\circ} \mathrm{C}$ 热处理除去催化剂组分 $\mathrm{NMe}_{3}$ 得到PDMS胶(图3). 合成的PDMS胶能在氯仿及四氢呋喃(THF)中产生定 向作用, 随后利用 $\mathrm{RDC}$ 在氯仿中测定了倍半萜类石竹 烯( $\beta-(-)$-caryophyllene) 的构象及有机亚膦酰胺与金属 钯络合物中间体的构型, 但是定向能力较弱 ${ }^{[2]}$.

Gil教授等 ${ }^{[33]}$ 最初发展的交联聚甲基丙烯酸甲酯 胶(PMMA)定向介质, 在对天然产物ludartin的绝对构 型分析中得到初步认定. PMMA在氯仿中的定向能 力也与其交联度成正比, 且采集图谱背景峰较弱. 由
于使用传统的自由溶胀制胶方法, PMMA胶的制备比 较费时(需20 30d), 测试分子均匀扩散进胶内也需要 2 3 d. 随后Gil教授等 ${ }^{[34]}$ 通过在核磁管内植入Shigemi 活塞, 加入氯仿后挤压胶来快速制备不同定向能力角 度的PMMA胶, 修正了制胶繁琐等缺点, 目前这种活 塞挤压装置已实现了商品化(图4) ${ }^{[35]}$; 另外, 通过溶剂 洗涤的方法实现对胶的回收, 这些优点无疑拓宽了其 适用性.

凝胶难于制备成手性定向介质,而众所周知的聚 谷氨酸芐酯(PBLG) 高分子具有螺旋结构. 为弥补这一 缺憾, 2013年, Thiele等 ${ }^{[36]}$ 在交联剂三乙烯四胺(TETA) 的作用下合成了具有螺旋结构的手性PBLG胶(图5). 可以实现对异松蒎醇(IPC)和樟脑磺内酰胺(Cs) 对映体 的区分, 其缺点是制胶及平衡过程需要较长时间 $\left(40^{\circ} \mathrm{C}\right.$ 下平衡 1 2月), 并且胶在应用过程中很容易碎裂.

\subsection{2 极性有机溶剂定向胶}

2005年, Griesinger教授等用单体2-丙烯酰胺-2-甲 基丙磺酸(AMPS) 与 $N, N$-二甲基丙烯酰胺(DMAA) 在 $N, N$-亚甲基双丙烯酰胺(BIS)和引发剂过硫酸铵(APS) 作用下发生聚合反应, 得到交联的聚丙烯酰胺共聚物 (PH胶或称PH-PDMAA胶)是第一个可溶于二甲基亚 砜(DMSO)的定向胶, 它也可以与水、 $N, N$-二甲基甲酰 胺(DMF)兼溶. PH胶的定向能力适中, 比PBLG定向能 力小; 氢谱线宽窄, 有利于 RDC的采集及分析 ${ }^{[37]}$.

2012年Reinscheid等 ${ }^{[38]}$ 通过修饰PH胶单体(AMPS), 采用 $(R)-\mathrm{APhES}$ 或 $(S)$-APhES为单体按照上述Griesinger

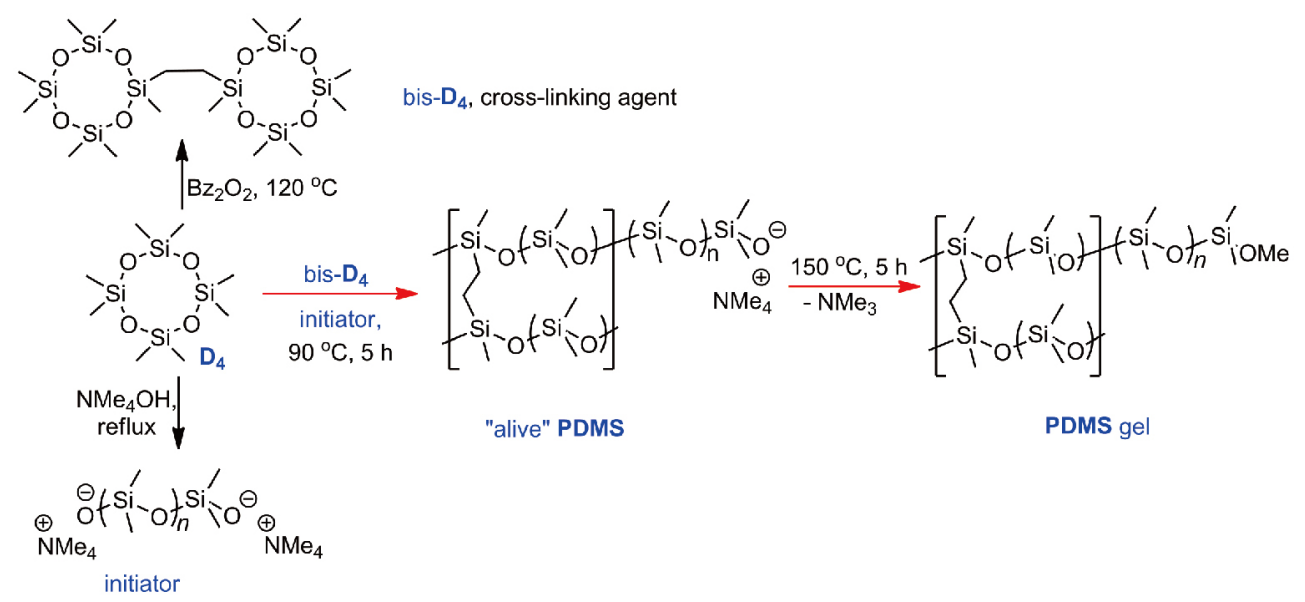

图 3 化学合成制备PDMS高分子胶 ${ }^{[32]}$ (网络版彩图) 

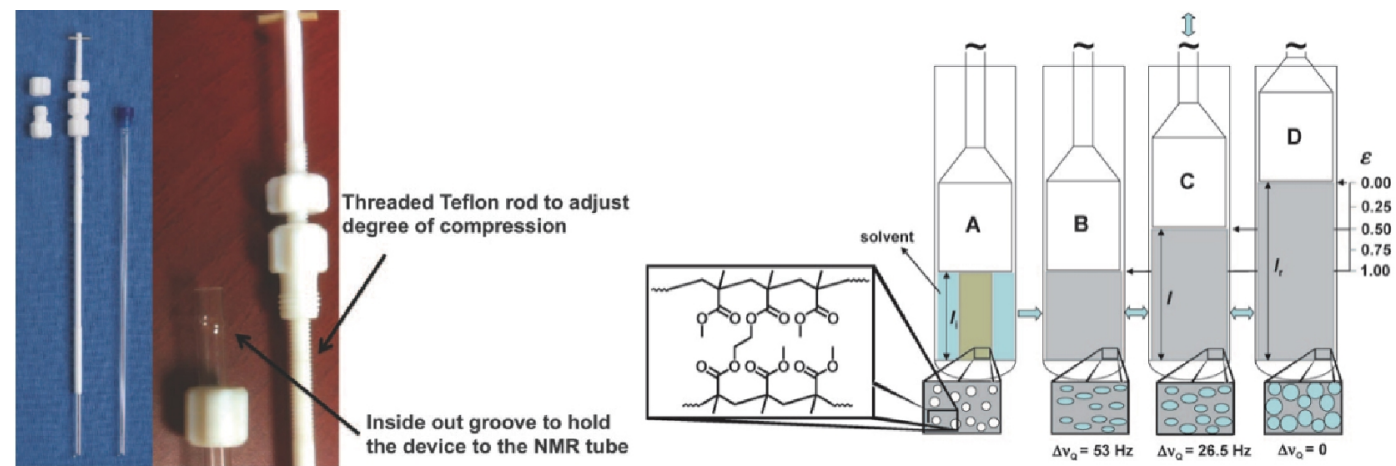

图 4 商品化的挤压装置 ${ }^{[33,35]}$ 和经定向调节的PMMA胶 ${ }^{[34]}$ (网络版彩图)

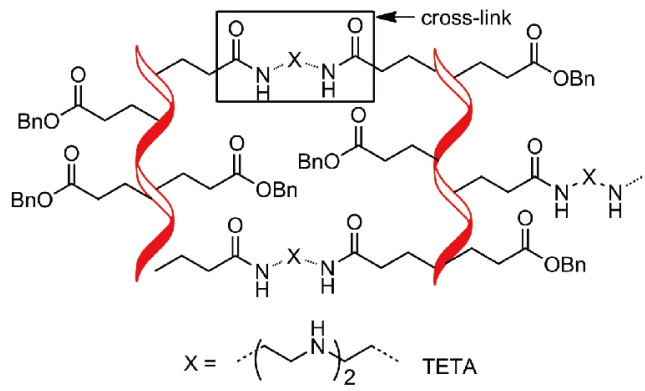

图 5 交联剂作用下产生的手性PBLG胶 ${ }^{[36]}$ (网络版彩图)

教授等的聚合方法, 发展了具有手性的 PH胶, 在 DMSO、甲醇和水溶剂中均适用, 能有效区分对映 体薄荷醇, 对映体盐酸甲氟喹和马钱子碱盐酸盐等有 机小分子(图6).

Luy等 ${ }^{[39]}$ 发展的聚醋酸乙烯酯(PVAc) 凝胶, 其合 成过程与PS胶类似. PVAc胶具有广泛的溶剂普适性, 尤其在极性有机溶剂中增加了其实用性. 通过对天 然产物降樟脑 norcamphor和抗生素 sphaeropsidin A 的 $\mathrm{RDC}$ 值分析, 验证了 PVAc胶作为定向介质的实际应用 价值.

通过电子能量激发丙烯腈产生交联的聚丙烯腈 凝胶(PAN) 可溶解于 DMSO和DMF中, 可以调控为合 适的定向排列 $\left(\Delta v_{\mathrm{Q}}=10 \sim 20 \mathrm{~Hz}\right)$. 由于PAN胶不带电, 可 以与多肽等分子相溶, 应用范围较广 ${ }^{[40]}$. 受全気代PS 胶启发, 2010年, Luy 等 ${ }^{[41]}$ 又发展了全氛代dPAN胶, 克 服了背景峰干扰的缺点, 为解析样品量少且蕴含 RDC 信息较少的天然产物结构提供了可能.

2013年, Luy等 ${ }^{[42]}$ 报道了一种新型交联聚乙二醇 胶(PEO). 根据合成方法可以将其分为两类: 一是利用 $\gamma$ 射线激发交联的 $\gamma$-PEO凝胶; 另一种是化学修饰聚合
的PEOMMA胶. PEO能溶于水和各种有机溶剂以及混 合溶剂中, 其广泛的溶解性几乎成为一种“万能的”定 向胶. PEO胶也存在一些缺点: 在强酸环境下会变性; 高于 $50^{\circ} \mathrm{C}$ 下会软化变质. 另外, $\mathrm{PEO}$ 胶不具有手性, 因 此在进行对映异构体区分时需要加入足量的手性加 合物才能实现(图7).

最近, Gil教授等 ${ }^{[43,44]}$ 发展了聚甲基丙烯酸着全乙酯胶 (p-HEMA)和聚乙二醇甲醚甲酰酯胶 (p-DEGMEMA), 其物理特性及制备过程与PMMA胶类似, 是另外两种 新型的、分别溶于DMSO和甲醇的极性凝胶定向介质。 在采用JSB-HSQC实验对柔性分子薄荷醇(Menthol)及 天然产物倒千里光碱(Retrorsine)采集数据, 得到了质 量更高、数据更优的图谱.
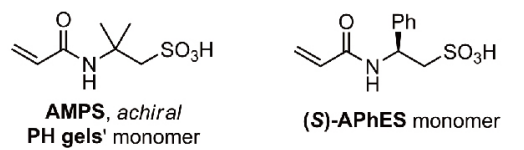

(S)-APhES monomer<smiles>C=CC(=O)NC(CO)[PH](=O)c1ccccc1</smiles>

(R)-APhES monomer (a)

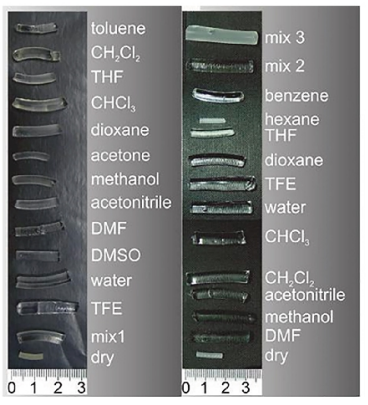

(b)

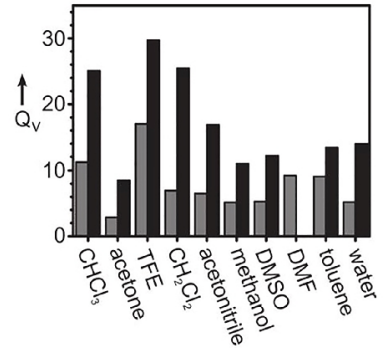

图 7 两种交联的PEO在各种有机溶剂中溶胀产生的胶及 相应的 ${ }^{2} \mathrm{H}$ 四极裂分 $\Delta v_{Q}{ }^{[42]}$ (网络版彩图) 


\subsection{3 水溶性定向胶}

水溶性聚丙烯酰胺水凝胶(PAA) 是最早应用于测 定生物大分子结构的定向凝胶, 在核磁管内通过压缩 或拉伸进行定向角度调节 ${ }^{[45,46]} .2005$ 年Luy教授等 ${ }^{[4]}$ 发现, 一种含有明胶的德国糖果(Gummibärchen)在经 过简单处理纯化后, 经溶胀配制, 四极㲴谱 $\Delta v_{\mathrm{Q}}$ 能产生 $21 \mathrm{~Hz}$ 的裂分. 受此启发, 随后对日常生活用的明胶 (gelatin)进行研究, 发现明胶不仅可以作为弱定向介质 确定分子的相对构型, 还可以作为手性定向介质区分 对映异构体. 随后, 通过电子加速使明胶交联成带负 电荷的e-gelatin也可以作为手性定向介质, 利用RDC或 RQCs (残留四极耦合) 等各向异性参数可以对丙氨酸 对映体有效识别 ${ }^{[48]}$. 与明胶类似, 胶原蛋白(collagen) 聚合物也可作为有效区分氨基酸异构体和测定生物 大分子谷氨酰胺结合蛋白质 $\mathrm{GlnBP}$ 的各向异性参数的 定向介质 ${ }^{[49,50]}$.

表1 详细总结了近年来发展的在不同溶剂中定向 有机小分子的凝胶介质及其性质.

\section{2 溶致液晶}

液晶是一种介于液态与结晶态之间的物质状态, 具有液体流动性和晶体各向异性的性质, 在很多生物 体系中都能观察到液晶, 如脂质、纤维素和DNA都能
形成液晶相 ${ }^{[51]}$. 根据生成环境的条件不同可把液晶分 为两大类: 一是在某一温度范围内显现的液晶相物质 称为热致液晶; 二是某些物质溶解于水或有机溶剂后 在某一浓度下呈现的液晶相称为溶致液晶. 溶致液晶 的形貌与溶液的浓度、温度、 $\mathrm{pH}$ 和离子强度均有一 定关系. 根据分子排列的方式, 液晶也可分为近晶相 液晶、向列相液晶和胆甾相液晶等 ${ }^{[2]}$. 与凝胶相比, 液晶具有一个最重要的优点: 它制样迅速, 平衡时间 短, 因而受到广泛关注, 从而拓宽了定向介质发展, 为 $\mathrm{RDC}$ 的提取以及分子结构鉴定发挥了重要的作用. 根 据溶致液晶的组成和性质, 可以将液晶定向介质分为 以下几种类型.

\subsection{1 聚多肽类液晶}

早在1954年, Doty等 ${ }^{[53]}$ 发现, 聚 $L$-谷氨酸 $\gamma$-市酯 (PBLG) 可以形成向列相液晶. 随后法国 Courtieu和 Lesot教授等 ${ }^{[54-60]}$ 利用PBLG的定向作用在非极性有机 溶剂中使用同位素标记分析物或気谱实验等方法研究 有机小分子异构体识别与区分, 使PBLG的应用蓬勃 发展. 2003年, Merlet, Thiele和Griesinger ${ }^{[61163]} 3$ 个独立 课题组几乎同时报道了在PBLG中测定小分子的相对 构型, 成功开辟了 RDC在有机分子及天然产物的立体 结构解析方面的广阔天地, 具有里程碑式意义. 随后 更多的聚多肽 $\alpha$-螺旋高分子被用作弱定向介质, 主要

表 1 可拉伸的凝胶定向介质溶剂的兼容性

\begin{tabular}{|c|c|c|}
\hline 凝胶 & 共存溶剂种类 & 手征性 \\
\hline Gelatin & $\mathrm{D}_{2} \mathrm{O}$ & $\sqrt{ }$ \\
\hline $\mathrm{e}^{-}$-Gelatin & $\mathrm{D}_{2} \mathrm{O}, \mathrm{DMSO}$ & $\sqrt{ }$ \\
\hline Collagen & $\mathrm{D}_{2} \mathrm{O}$ & $\sqrt{ }$ \\
\hline PAA & $\mathrm{D}_{2} \mathrm{O}$ & $\times$ \\
\hline $\mathrm{PH}$ & $\mathrm{D}_{2} \mathrm{O}, \mathrm{DMSO}, \mathrm{DMF}$ & $\times$ \\
\hline APhES-PH & $\mathrm{D}_{2} \mathrm{O}, \mathrm{DMSO}, \mathrm{CH}_{3} \mathrm{OH}$ & $\sqrt{ }$ \\
\hline PAN, dPAN & DMSO, DMF & $\times$ \\
\hline PEO & $\mathrm{D}_{2} \mathrm{O}, \mathrm{CD}_{3} \mathrm{CN}, \mathrm{CD}_{3} \mathrm{OH}, \mathrm{DMSO}$, DMAC, Acetone, THF, $\mathrm{CDCl}_{3}, \mathrm{CD}_{2} \mathrm{Cl}_{2}, \mathrm{C}_{6} \mathrm{D}_{6}$, Dioxane, $n$-Hexane & $\times$ \\
\hline PVAc & $\mathrm{CDCl}_{3}, \mathrm{CD}_{2} \mathrm{Cl}_{2}, \mathrm{C}_{6} \mathrm{D}_{6}, \mathrm{CD}_{3} \mathrm{OH}, \mathrm{CD}_{3} \mathrm{CN}$, DMSO, DMF, Acetone, EtOAc, Dioxane & $\times$ \\
\hline PMMA & $\mathrm{CDCl}_{3}, \mathrm{CD}_{2} \mathrm{Cl}_{2}, \mathrm{C}_{6} \mathrm{D}_{6}, \mathrm{CD}_{3} \mathrm{CN}$, Acetone, EtOAc & $\times$ \\
\hline PS, dPS & $\mathrm{CDCl}_{3}, \mathrm{CD}_{2} \mathrm{Cl}_{2}$, THF, $\mathrm{C}_{6} \mathrm{D}_{6}$, Dioxane & $\times$ \\
\hline PDMS & $\mathrm{CDCl}_{3}, \mathrm{CD}_{2} \mathrm{Cl}_{2}, \mathrm{THF}, \mathrm{C}_{6} \mathrm{D}_{6}$, Dioxane, $n$-Hexane & $\times$ \\
\hline PBLG gel & $\mathrm{CDCl}_{3}, \mathrm{CD}_{2} \mathrm{Cl}_{2}$, THF, $\mathrm{C}_{6} \mathrm{D}_{6}$, Dioxane & $\sqrt{ }$ \\
\hline p-HEMA & DMSO & $x$ \\
\hline p-DEGMEMA & $\mathrm{CD}_{3} \mathrm{OH}$ & $x$ \\
\hline
\end{tabular}


包括PBLG 的对映体PBDG, PCBLL/PCBDL和PELG/ PEDG等. 由于 $P B L G$ 定向能力太强使耦合复杂, 造成 图谱解析困难等缺点, 之后, Thiele教授等对此类液 晶进行了更加详细的研究, 主要集中在降低其定向能 力、改变溶质取向以及加强对映异构体识别能力方 面, 目前PBLG液晶已发展为商品化的定向介质(图8). 通过增加聚合物的分子量, 使引入的聚合物链增长, PBLG 能表现出更加均匀的液晶相, 同时降低了定向 强度, 增强其适用性, 即使在临界浓度低于 $10 \mathrm{wt} \%$ 时 也可以产生很好的图谱. 这对于研究其他多肽共聚物 的定向性能, 甚至其他类型高分子液晶形成机制提供 了理论基础 ${ }^{[6470]}$.

相比聚多肽二级结构的 $\alpha$-螺旋而言, $\beta$-聚多肽可 以以更短的链长度形成液晶, 且临界浓度更低, 定向 能力也相对弱一些, 更适合小分子RDC提取. 2011年, Thiele教授等 ${ }^{[71]}$ 发展了聚 $\beta-\mathrm{ACH}-$ 多肽液晶. 此液晶在 水中的临界浓度可低至 $1 \mathrm{wt} \%$. 通过对脯氨酸的 RDC
分析, 验证了聚 $\beta$-ACHC-多肽液晶作为定向介质的准 确性和适用性. 此外在对映异构体识别能力方面表现 显著(图9).

本课题组 ${ }^{[72]}$ 最新发展的AAKLVFF多肽液晶(图10), 在低浓度的甲醇溶剂中能自组装成液晶, 也是首例 甲醇体系中的液晶介质, 定向底物广谱. 具有不同 官能团或芳香结构的Artemether, Ingenol, Gibberellin, Ginkgolide B, Actinomycin D, aristolochic acid A, indole3-acetic acid等天然分子已被成功检测, 且底物提取相 关RDC值的大小能通过液晶溶度的调节, 其低浓度、 低黏度、易制备的特点, 增加了AAKLVFF多肽液晶 的实用性 ${ }^{[72]}$, 它可以通过经典的固相合成实现商品化, 具有广泛的应用前景.

\subsection{2 螺旋手性高分子液晶}

2010年, Reggelin教授 ${ }^{[73]}$ 报道了聚苯乙基胍 $((R)-$ PPEMG)液晶, 其可在 $18.7 \mathrm{wt} \%$ 的氯仿溶液中形成弱定
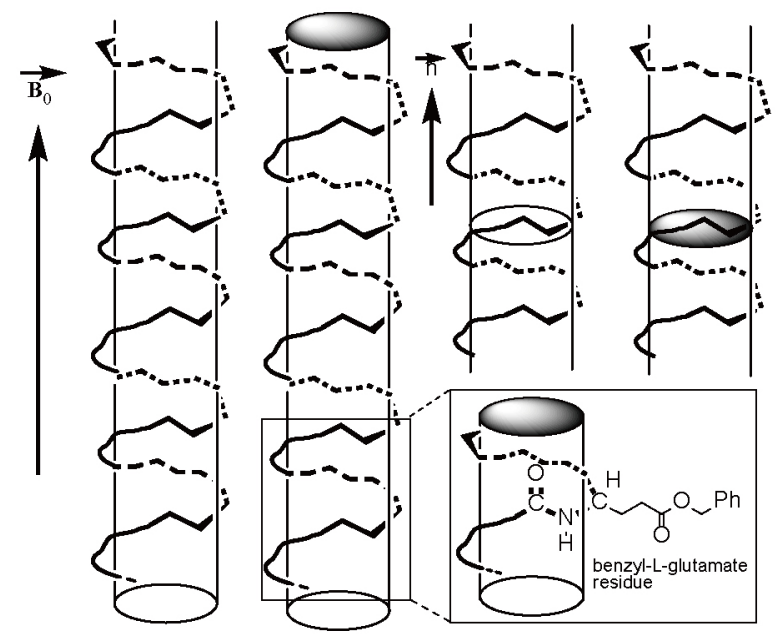

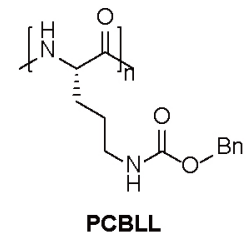<smiles>[R]OC(=O)CC[C@H](NC)C(=O)O</smiles>

$\mathrm{R}=\mathrm{Bn} \cdot \mathrm{PBLG}$ $\mathrm{R}=\mathrm{Et} ;$ PELG

Homopolypeptide

图 8 PBLG的 $\alpha$-螺旋二级结构示意图及聚多肽分子结构 ${ }^{[69]}$

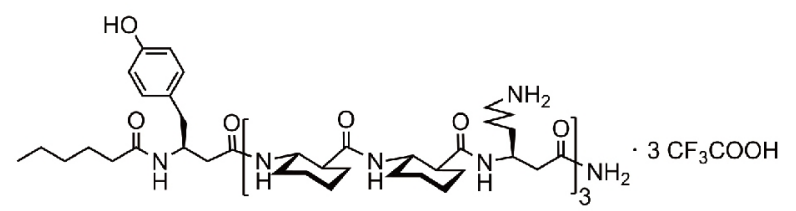

ACHC-rich $\beta$-peptide

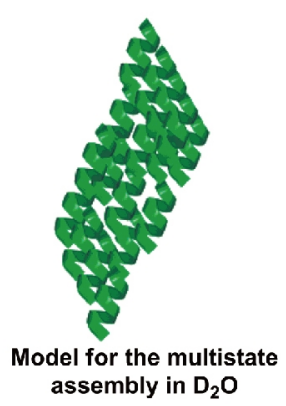

assembly in $D_{2} \mathrm{O}$

图 $9 \beta-\mathrm{ACH}-$ 多肽结构及液晶自组装模型 ${ }^{[71]}$ (网络版彩图) 


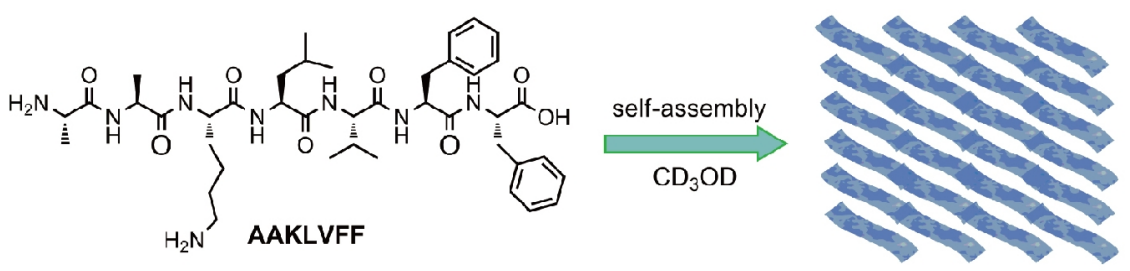

图 10 AAKLVFF多肽结构及在甲醇中的自组装模型 ${ }^{[72]}$ (网络版彩图)

向的液晶, 且能够实现对 IPC 的对映异构体区分. 然 而高浓度液晶会造成图谱半峰宽增加, 限制了对RDC 的准确分析; 定向强度虽然比PBLG液晶小很多, 但 仍然达到 $1000 \mathrm{~Hz}$, 耦合作用过强; 样品制备过程中纯 化未形成液晶的高分子或未聚合的单体过程太繁琐 (图11). 为寻找一种能代替聚胍的定向介质,一方面保 留聚胍单手螺旋结构的优点, 另一方面使定向能力更 弱, 更适宜提取RDC. 2012年, Reggelin等 ${ }^{[74,75]}$ 和Berger 等 ${ }^{[76]}$ 分别发展了手性氨基酸正癸酯类乙炔定向介质, 它们的长链结构以及很高的分子量使其在较低的浓 度下就能形成液晶相, 降低了液晶的临界浓度. RDC 值在 $-10 \sim+15 \mathrm{~Hz}$ 合适的范围内, 且峰形窄, 大大提高 了提取 $\mathrm{RDC}$ 的准确度.

2012年, Berger等 ${ }^{[77]}$ 按照相似的思路合成了丙氨 酸正癸酯聚异腈高聚物, 聚异腈在氯仿、THF、二氯 甲烷中均能形成液晶, 在此液晶介质中的待测分子马 钱子碱能呈现较弱的定向排列, 但没有报道其手性识 别性能(图12). 2017年, Reggelin教授 ${ }^{[78]}$ 通过热处理的 方法对此高分子液晶进行优化, 热处理后液晶性能更 加稳定, 并成功应用于区分对映体IPC. 在此之前, 本 课题组 ${ }^{[79,80]}$ 已经相继合成出了轱氨酸正癸酯以及苯丙 氨酸芐酯为侧链的螺旋聚异腈高聚物弱定向介质, 通 过采集相关残留偶极耦合值表明这类氨基酸类螺旋 聚异腈高分子在手性模型化合物对映体区分方面都 具有较好的识别性能(图12).

本课题组 ${ }^{[81]}$ 还发展了一类新型二肽类聚异腈液 晶 $(L, L$-PIAF-OBn)弱定向介质, 该结构在刚性异腈主 链的螺旋外力作用下自组装为有序聚集体, 且肽链侧 基的引入产生的氢键作用可稳定这种生物模拟的螺 旋优势构象, 为构筑这种新型的定向介质提供协同效 应, 它可在较低浓度的氯仿溶液中自成液晶相. 这一 特征被成功用于马钱子碱、雷公藤甲素等复杂天然 分子的立体化学研究; RDC计算结果与其单晶原子坐 标的空间构型相吻合, 且获得的图谱高分辨(图13).<smiles>CC(=NC(C)c1ccccc1)N(C)C</smiles>

(R)-PPEMG

Polyguanidine

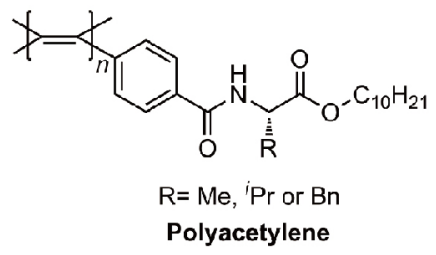

Polyacetylene
图 11 手性螺旋聚胍和聚乙炔骨架高分子结构 ${ }^{[73 ~ 76]}$

2017 年, Thiele教授等 ${ }^{[82]}$ 采用 $C_{3}$ 对称的正十二烷 基及手性癸烷基取代的苯三甲酰胺(BTA), 利用其分 子结构特殊的几何构型通过氢键和 $\pi-\pi$ 堆积作用将分 子扩展为三维超分子结构(图14), 其非手性正十二烷 基苯三酰胺聚合物可在弱极性溶剂 $\mathrm{CDCl}_{3}$ 和 $\mathrm{CCl}_{4}$ 中组 装成液晶相(临界浓度分别为 $26.0 \mathrm{wt} \%$ 和 $14.9 \mathrm{wt} \%$ ), 可 以准确采集双环戊二烯小分子的RDC值. 而手性癸烷 苯三酰胺螺旋聚合物单独在有机溶剂中不能形成液 晶相, 但与非手性正十二烷基苯三酰胺组成的共聚物 存在下会产生手性放大效应(该现象在共聚物超分子 化学中称为典型的“军官-士兵效应”, 即 $\mathrm{SaS}$ ), 它们能 在 $\mathrm{CDCl}_{3}$ 和 THF- $d_{8}$ 中组装为手性螺旋液晶, 据此他们 研究了对映体 $\beta$-蒎烯在此液晶中的手性区分性能 ${ }^{[82]}$.

\subsection{3 氧化石墨烯液晶}

氧化石墨烯(GO)作为近年来科学界的研究热点, 因其特性的理化性质: 结构对称、刚性大、纵横比高 和超高分子量, 可在 $1 \mathrm{mg} / \mathrm{mL}$ 浓度下形成液晶相. 2014 年, 本课题组 ${ }^{[83}$ 将GO液晶作为弱定向介质用于有机小 分子的RDC的提取分析, 相较于上述高分子聚合物液 晶(PBLG、聚异腈、聚乙炔等), GO液晶配制过程更 加快捷简单, 只需将待测分子与适量氧化石墨烯以及 溶剂装入核磁管中, 摇晃混匀, 离心平衡后即可应用, 无需长时间平衡制备样品, 整个过程几分钟内即可完 成. 而且 GO具有较广的溶剂适用性, 既可以溶于水, 也 可溶于混合溶液, 如水-DMSO、水-乙腈、水-丙酮中. 此外, GO液晶性质非常稳定, 在 $\mathrm{pH}$ 1 14以及温度 5 $80^{\circ} \mathrm{C}$ 范围内均保持液晶相, 测得的谱图无背景峰, 这是 
<smiles>CCCCCCOC(=O)C(C)NC(=O)c1ccc(N=C(C)C)cc1</smiles>

L,L-PIAF-OBn

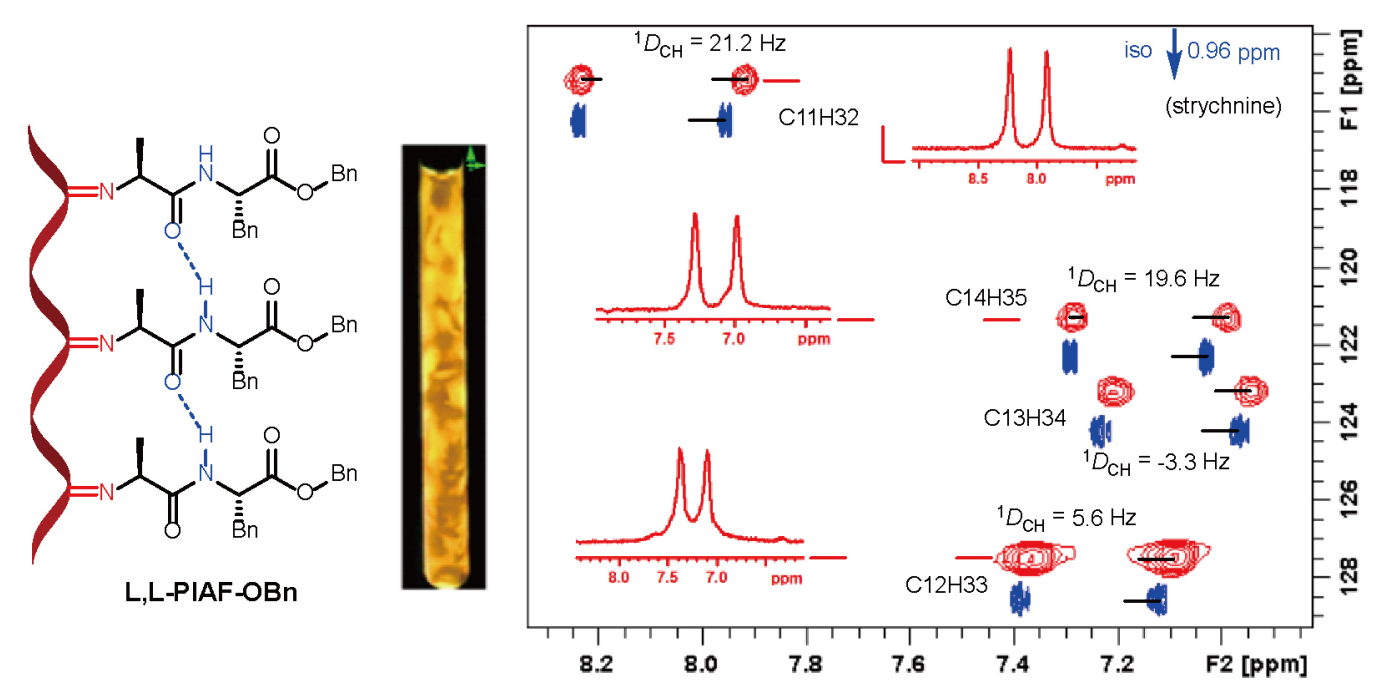<smiles></smiles><smiles>CC(C)=Nc1ccc(C(=O)NC(Cc2ccccc2)C(=O)OCc2ccccc2)cc1</smiles>

Polyisocyanide

图 12 手性螺旋聚异腈骨架高分子结构 ${ }^{[77 ~ 80]}$

图 13 螺旋二肽聚异腈的结构, 液晶图片及 $L, L-P I A F-O B n$ 定向介质采集马钱子碱局部二维图谱(蓝色: 各向同性的核磁信 号; 红色: 各向异性核磁信号 ${ }^{[81]}$ (网络版彩图)
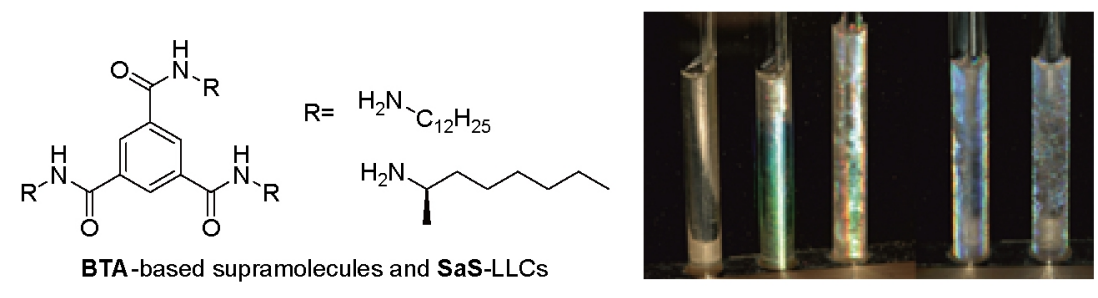

图 14 超分子BTA的分子结构及自组装的SaS-液晶图片 ${ }^{[82]}$ (网络版彩图)

其他定向介质所不具备的特征. GO定向能力适中, 提 取的 RDC值在适宜的范围内, 其 $\Delta v_{\mathrm{Q}}$ 随石墨烯液晶的 浓缩呈现规律性变化, 可以通过改变其浓度来调控其 定向能力(图15). 虽然 $\mathrm{GO}$ 作为定向介质具有如上众多 优点, 但其仍然存在一些瑕疵: 不能全溶于有机溶剂, 由于 $\pi-\pi$ 堆积效应的存在底物宽泛性受限, 黏度较高导 致线宽较宽.

为解决 $\mathrm{GO}$ 液晶在常规纯有机溶剂的定向排列问 题, 进一步探讨液晶形成机制及拓展其应用. 2016年, 本课题组 ${ }^{[84]}$ 通过自由聚合的方法把甲基丙烯酸三氟
乙酯(TFEMA)嫁接于 GO表面, 得到具有二维分子刷 结构的 GO- $g$-TFEMA, 增加了 GO 在纯DMSO 中的分 散性, 成功观察到液晶相行为, 并被 ${ }^{2} \mathrm{H} N \mathrm{NR}$ 实验和同 步辐射小角X射线散射(SAXS)技术所证实; 进而以抗 疮疾药物双氢青蒿素和雌素酮为模式分子, 进行RDC 的测定, 计算结果与其单晶原子坐标的空间构型相吻 合(图 16). 由于嫁接的氧化石墨烯液晶无背景信号, GO- $g$-TFEMA液晶定向介质目前受到德国 Griesinger 教授等 ${ }^{[85}$ 的青睐, 该液晶在研究雌性激素药物乙炔 雌二醇的构型、构象分析方面提供了一种新的定向方法. 

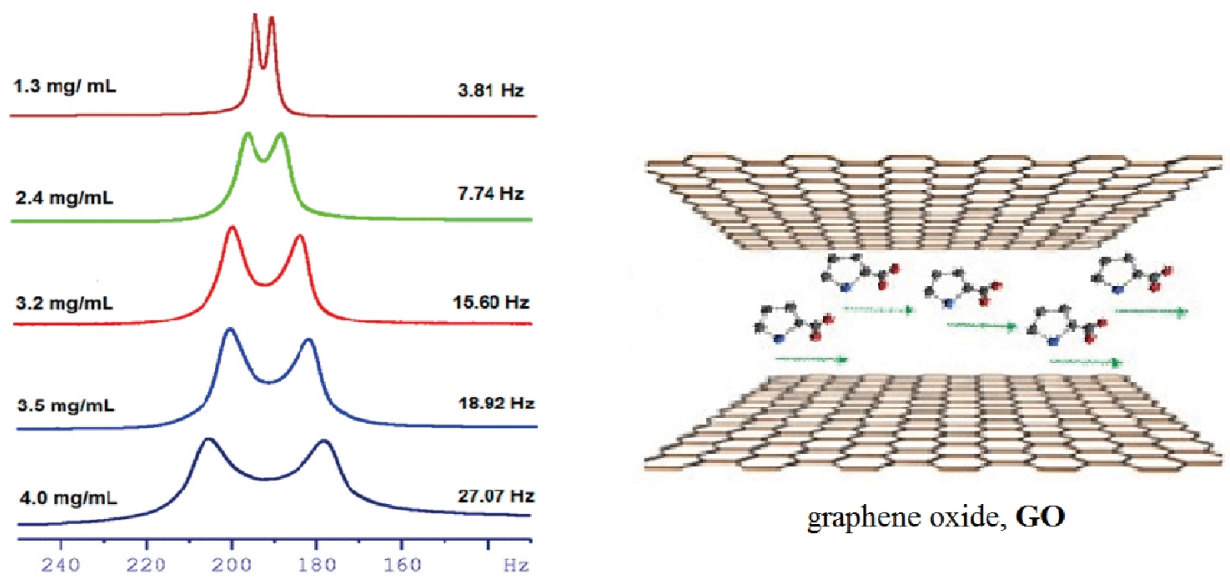

图 15 浓度可调的 GO液晶和有机分子在GO液晶中定向排列示意图 ${ }^{[83]}$ (网络版彩图)

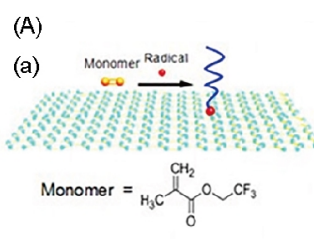

(b)

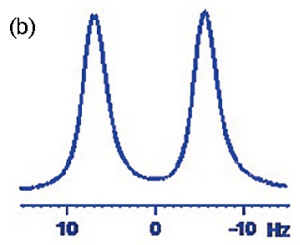

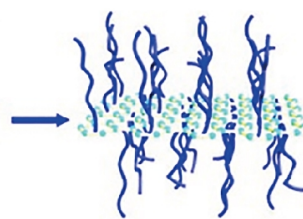

(c)

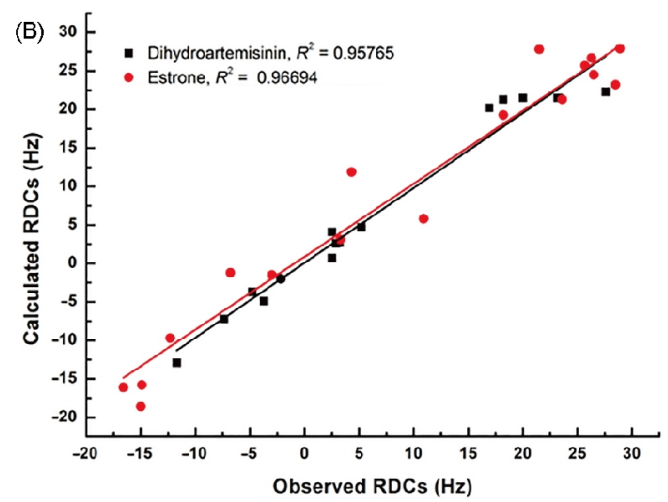

图 16 GO-g-TFEMA液晶制备相关图片(A)及双氢青蒿素和雌素酮RDC值的线性拟合关系(B). (a) 分子刷结构GO- $g$-TFEMA 的合成示意图; (b) ${ }^{2} \mathrm{H} N \mathrm{NR}$ 实验测得的四极氛谱裂分值 $\Delta v_{\mathrm{Q}}$; (c) 核磁管内的液晶图片 ${ }^{[84]}$ (网络版彩图)

\subsection{4 其他水溶性液晶}

早在20年前, 棒状或纤维状的花叶病毒(TMV)、 噬菌体(Pf1 phage)、圆盘状磷脂双分子层(bicelle)胶粒 及著名的表面活性剂/醇体系形成的Otting介质相, 被发 现在水溶液体系中具有定向作用, 随后广泛应用于生 物大分子的结构测定. 但是由于这些液晶对有机小分 子的信号干扰太大而鲜有用于小分子结构的测定 ${ }^{[86,87]}$.

兼溶于水和DMSO的五甘醇单十二烷基醚 $\left(\mathrm{C}_{12} \mathrm{E}_{5}\right)$ 液晶, 其定向能力可以通过调节温度和浓度来改变, 可以适用于测定小极性有机分子氨基酸和五环内酯 的残留偶极耦合值(图 17) ${ }^{[88,89]}$.

2013 年Berger等 ${ }^{[90]}$ 利用溴化 $N$-十二烷基 $-N$-甲基 吡咯烷鎓盐 $\left(\mathrm{C}_{12} \mathrm{MPB}\right)$ 离子型在水、醇或DMSO形成的 三元体系液晶应用于测定葡萄糖RDC值(图 17), 以及
最近经上述吡咯盐结构改造的咪唑类的离子型液晶 $\left(\mathrm{C}_{12} \mathrm{MImBF}_{4}\right)$, 该液晶用于茶碱有机分子的同核RDC值 测定 ${ }^{[91]}$.

2014年Navarro-Vázquez教授等 ${ }^{[92]}$ 发现弱定向介质 色甘酸二钠(DSCG), 成功提取水溶性有机小分子的残 留偶极耦合值, 并且通过改变浓度、温度和加入盐水 3 种方法调节定向强度. 加入盐水可减弱介质的定向 程度, 使残留偶极耦合值落在易控制的范围内, 且可 以通过控制温度在同一样品中采集到各向同性和各 向异性的RDC值(图 17).

2013年Suryaprakash教授等 ${ }^{[93]}$ 发展了一种新的核 苷酸单磷酸盐手性液晶, 该弱定向介质利用残留偶 极耦合参数用于水溶性的脯氨酸异构体区分及三肽 (Gly-Glu-Cys)化合物结构的测定(图18). 


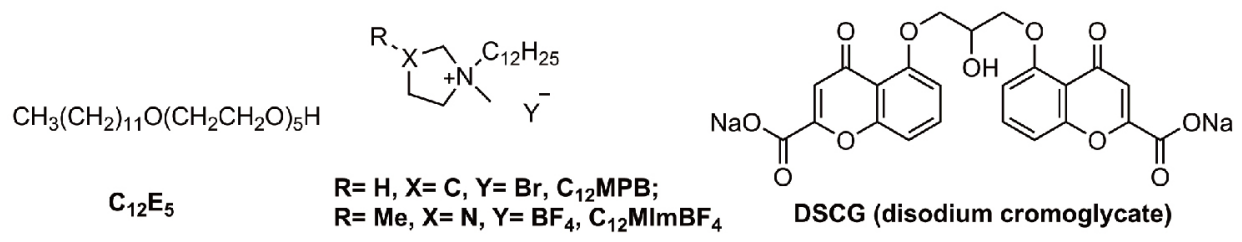

图 17 水溶性液晶介质结构 ${ }^{[8-9-92]}$
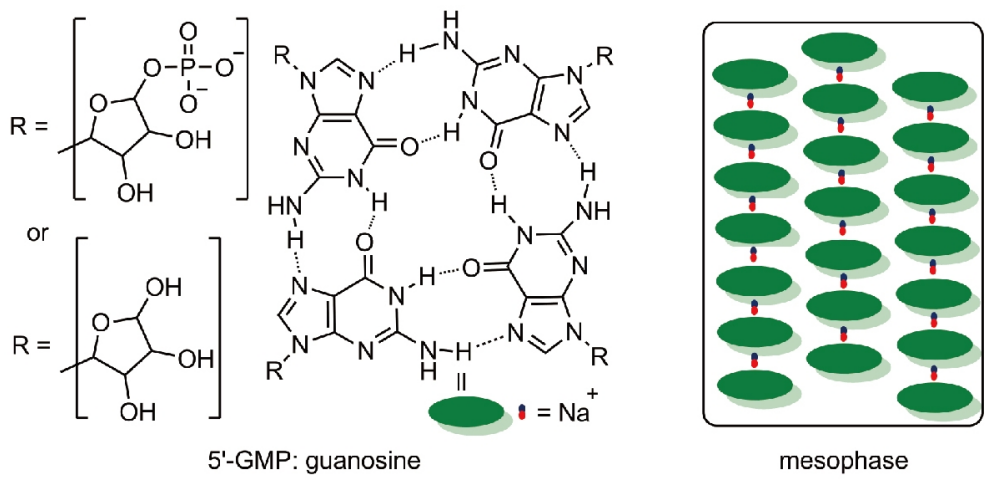

mesophase

图 18 核苷酸单磷酸盐(5'-GMP: guanosine)结构及液晶模型 ${ }^{[93]}$ (网络版彩图)

\section{RDC测定有机立体化学分析方法的构建}

$\mathrm{RDC}$ 的提取是通过核磁共振二维实验获得的, 一 般测定同核或异核: 如C-H, C-C, N-H和H-H核之间的 残留耦合值, 耦合值可以测定单键或者远程耦合值 $\left({ }^{n} D\right.$, $n=1,2, \ldots)$. 原则上, 大多数二维 NMR脉冲实验技术可 以用于测量提取偶极耦合标量耦合. 然而, 由于样品 置于定向介质后, 会使 $T_{2}$ 缩小, 尤其是黏度较大的液晶 体系中, 使得线宽增加, 降低灵敏度, 同时也影响核磁 共振实验的演化延迟. 与同位素标记生物大分子不同, 有机分子, 尤其是天然产物分子的样品量有限, 为了提 高灵敏度和精度, Luy, Thiele, Bax以及 Griesinger等都 致力于发展核磁共振脉冲技术应用于测定有机小分 子和天然产物的各向异性参数, 表 2 简单列出了部分用 于RDC提取分析实验的各种脉冲序列及特点 ${ }^{[4-111]}$. 由 于在弱的定向介质中, 同核偕耦合及远程耦合的RDC 值太小, 精度不够, 在实际应用中有局限, 这里不作 主要介绍. 目前最受欢迎的是一键C-H RDCs, 其测 定方法主要是异核单量子相干实验 (HSQC), 常用的 序列为Luy教授等发展的纯同相的脉冲(clean inphase, CLIP)和纯反相的脉冲 (clean antiphase, CLAP). 常规的 $\mathrm{F}_{2}$-coupled HSQC 由于非灵敏核间的不匹配性产生弛 1410
豫延迟(INEPT)而造成较强的色散反相性, 往往会使信 号峰变形, 灵敏度低, 谱图质量达不到要求(图19(a)). 若在常规 $H S Q C$ 脉冲序列的末期施加一个 ${ }^{13} \mathrm{C}$ 轴的 $90^{\circ}$ 脉冲用于补偿相应的延迟信号, 即施加一个CLIP, 可 以得到清晰的谱图(图19(b)), 对于重叠的信号, 使用纯 反相的脉冲序列(clean antiphase, CLAP)也可使信号加 强, 图谱简化, 能得到较好的实验结果, 为了提高精度, $F_{2}$ 轴的点数在 $4 \mathrm{k}$ 以上为宜 ${ }^{[94]}$.

但如果分子中有较多的 $\mathrm{H}-\mathrm{H}$ 存在时, 线宽会有 所增加, 分辨率可能会降低, 尤其对于像甾体类、三 萜类和复杂生物碱等含有较多的亚甲基情况下, 可 能会影响耦合增加导致谱线变宽, 不能精准地测定 RDC. 最近, Luy, Thiele和Gil教授 ${ }^{[112-116]}$ 修正脉冲采集 相位, 通过 $\mathrm{F}_{1} J$-scaled HSQC, $\mathrm{F}_{1} J$-scaled BIRD HSQC (JSB-HSQC)（图 20) 或者 homodecoupled $J$-resolved HSQC (HD- $J$-HSQC) 等脉冲实验获得更加完美的图谱.

$\mathrm{RDC}$ 值的计算应用以下原理: 选择上述已见报 道或新发展的、合适的定向介质(凝胶或液晶), 经 ${ }^{2} \mathrm{H} N M R$ 实验考察其空间定向排列能力后, 将待测化合 物扩散到定向介质中, 利用合适的核磁脉冲实验, 预先 在各向同性条件下测定待测化合物的标量耦合 $\left({ }^{n} J\right)$; 其 次,在各向异性条件下(即弱定向介质中) 测定标量耦 
表 2 用于测定RDC的NMR脉冲序列简况

\begin{tabular}{ccc}
\hline RDC & NMR脉冲实验 & 特点 \\
\hline${ }^{1} D_{\mathrm{C}-\mathrm{H}}$ & CLIP/CLAP-HSQC, CLIP-ASAP-HSQC, CLIP/CLAP-RESET, & 清晰 \\
& J-X-BIRD ${ }^{d, X}$-HSQC, non-uniform sampling (NUS) & 高信噪比 \\
${ }^{1} D_{\mathrm{C}-\mathrm{H}},{ }^{2} D_{\mathrm{H}-\mathrm{H}}$ & E.COSY, P.E.HSQC & 单个实验 \\
${ }^{1} D_{\mathrm{C}-\mathrm{C}}$ & INADEQUATE, IP/AP-INADEQUATE & ${ }^{13} \mathrm{C}$ 丰度低, 需高浓度 \\
${ }^{n} D_{\mathrm{C}-\mathrm{H}}$ & HETLOC, XLOC & 不灵敏, 准确度高 \\
\hline
\end{tabular}
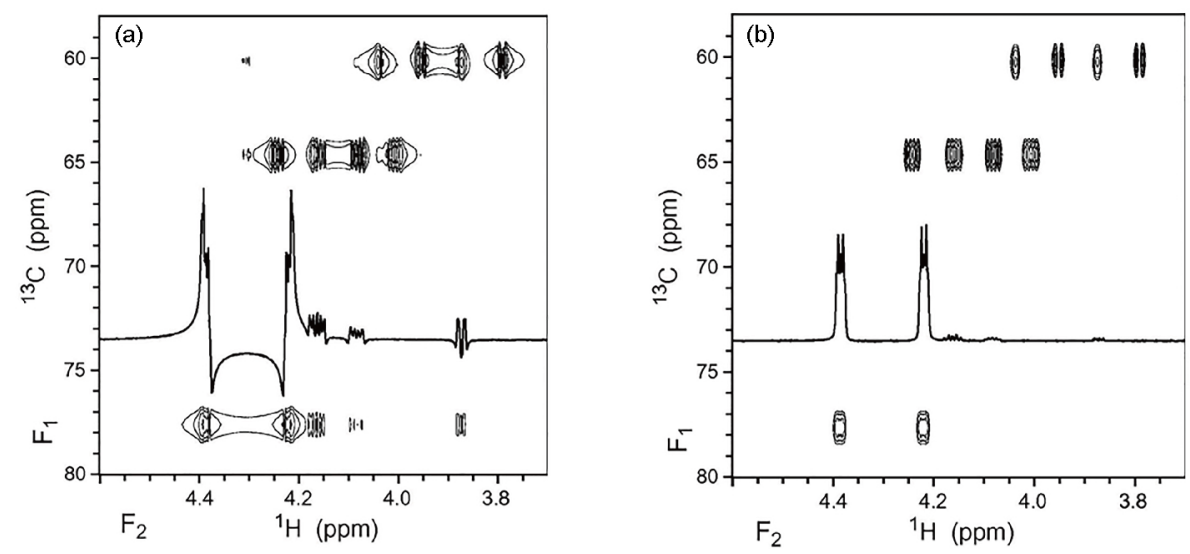

图 19 常规 $F_{2}$-coupled HSQC 实验(a)和CLIP-HSQC实验采集图谱(b)核磁信号比较

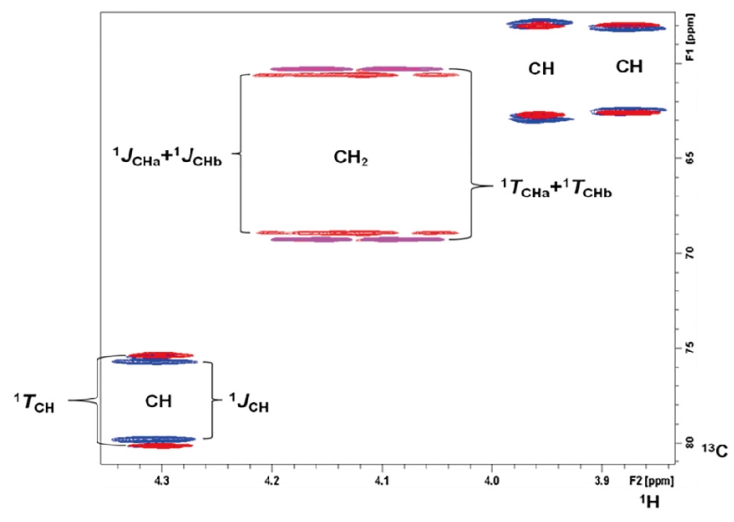

图 $20 \mathrm{~F}_{1}$ JSB-HSQC采集亚甲基和次甲基RDC示意图 ${ }^{[15]}$ (网 络版彩图)

合和偶极耦合的总和(即复合耦合值, ${ }^{n} T$ ), 残留偶极耦 合由两者的差值得到 $\left({ }^{n} D={ }^{n} T-{ }^{n} J\right.$, 一般测量 5 个以上正 交 $^{n} D$ 值, 越多越好). 通过合适的软件计算实验提取的 RDC与预测可能构型的RDC匹配性(SVD法拟合预测), 拟合结果一般通过 $Q$ 值反映出来 $\left(Q\right.$ 值为 ${ }^{n} D$ 实验测定值 和预测值的均方根, 最早由Cornilescu提出在液晶介质 中验证蛋白结构有效性), $Q$ 值越低, 说明建模预测的 构型与其实际构型一致, 从而建立运用RDC分析有机
小分子相对构型的方法(图21). 对于无法确定其构型 的合成分子或天然产物, 运用上述模型方法的流程, 通 过RDC提取分析, 或结合NOE相关谱、化合物的圆二 色谱(CD)或旋光色谱(ORD)、密度泛函理论(density functional theory, DFT)计算等必要的三维模型构建手 段, 就能清晰地给出化合物的构型(相对构型、构象, 甚至绝对构型 $)^{[117 ~ 121]}$.

利用残留偶极耦合参数识别与区分对映体的步 骤为: 按照上述RDC值计算流程图, 将手性对映体分 子分别在手性液晶检测上述NMR实验, 测定相应的 $\mathrm{RDC}$ 值, 然后将两个体系RDC值计算所得的定向排列 张量按以下公式计算:

$$
\begin{aligned}
& G C B=\cos \beta=\frac{\left\langle A^{(1)} \mid A^{(2)}\right\rangle}{\left|A^{(1)}\right|\left|A^{(2)}\right|} \\
& \text { with }\left\langle A^{(1)} \mid A^{(2)}\right\rangle=\sum_{i, j} A_{i j}^{(1)} A_{i j}^{(2)} \\
& \text { and }|A|=\sqrt{\langle A \mid A\rangle}=\sqrt{\sum_{i . j} A_{i j}^{2}}
\end{aligned}
$$

其中 $A$ 代表定向排列张量, $|A|$ 代表归一化的标量张量, GCB (the generalized cosine $\beta$ ) 为两个体系归一化标量 


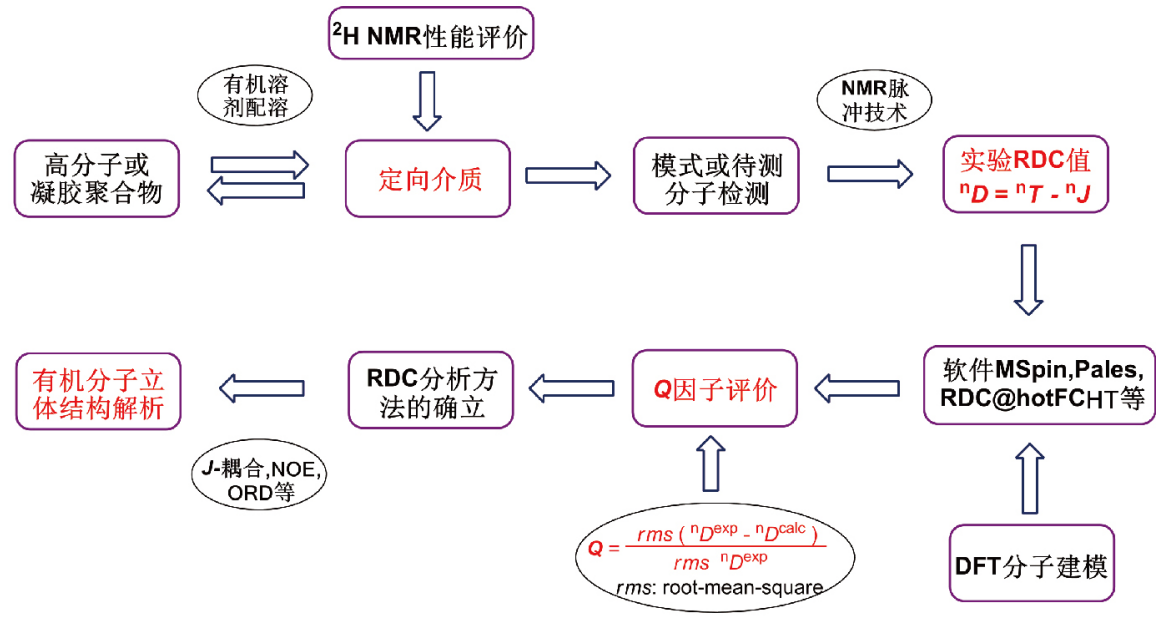

图 $21 \mathrm{RDC}$ 分析确认立体构型流程图(网络版彩图)

张量的非相似性, 即该值越大, $\beta$ 角度越小, 差异越小; 反之, 该值越小, $\beta$ 角度越大, 差异越大. 该方法早期主 要用在蛋白的动力学研究中, 现引入到手性区分能力 评价. 当一对手性对映体分别检测得到两套RDC值, 进行计算, 若得到 $\beta$ 角度越大时, 表示对映体在手性介 质的作用下, 在磁场中的方位差异越大, 即体现该定 向介质手性区分能力越强. 手性识别模型的构建, 从 理论上和实验上深入研究手性识别机制, 为 RDC确立 绝对构型奠定基础 ${ }^{[122 \sim 124]}$.

\section{RDC在天然产物结构鉴定中的应用}

残留偶极耦合已经在生物大分子如多糖、多肽、 蛋白质以及核酸的结构鉴定等方面得到了成功应用, 目前也成功应用于许多有机小分子, 其测定范围十分 广泛, 从简单结构分子到非常复杂分子, 从刚性分子 到柔性分子陆续被报道, 尤其是近些年在多手性中心 且灵活度高的天然产物绝对构型的测定方面有进一 步新发展. 本节全面汇总了近14年内(2003 2017年)利 用RDC测定天然产物结构的实例(图22及表3), 包括利 用 RDC对天然产物进行构象、相对构型和绝对构型 的分析测定.

\section{RDC 在有机合成结构分析中的应用}

除在天然产物结构鉴定之外, 利用RDC技术开展 有机合成化学立体化学结构的研究或反应机理中的 络合物中间体优势构象的研究已经取得了一定成果,
如具有不同生理活性的合成药物分子的相对构型或 绝对构型的测定; 涉及不同的有机反应类型: 光控反 应产生的不同构象形式; 不对称烯丙基烷基化中的金 属-配体络合物构象的研究; 过渡金属催化的Michael 加成反应产物绝对构型的测定等(图23和表4).

\section{RDC 在手性对映体区分中的应用}

发展具有手性的定向介质对于拓展RDC各向异 性参数的应用领域具有重要价值, 如手性小分子异 构体的区别与分析. 目前, PBLG/PBDG, PELG/PEDG, PCBLL/PCBDL等均聚多肽定向介质是研究对映体识 别使用最广泛的手性液晶, 早期的研究基本上是利用 氛或 ${ }^{13} \mathrm{C}$ 的天然丰度来区分对映异构体, 该方法需要样 品量较多, 且需耗费较长的采样时间, 后来侧重研究 $\mathrm{RDC}$ 各向异性参数在手性介质中的差异分析. 目前, 对于手性异构体的研究仍然处于不断寻求和发展优 秀的手性定向介质的阶段,即一般使用较为常见的刚 性结构的对映体模式分子(如IPC)作为分析物考察手 性介质的区分性能,而对于水溶性的定向介质一般使 用 $L$-或 $D$-丙氨酸或脯氨酸等天然氨基酸作为研究对 象(图24).

2013年, Thiele等 ${ }^{[36]}$ 利用聚合的PBLG聚合胶定向 介质, 通过 $\mathrm{F}_{1} \mathrm{SB}-\mathrm{HSQC}$ 实验记录异构体IPC和 $\mathrm{Cs}$ 二维 谱, 计算它们的一键C-H残留偶极耦合值差别在 $7 \mathrm{~Hz}$ 以 上, 其中映对体IPC区分角度达 $27^{\circ}$, 而在PBLG液晶中 的区分角度仅为 $8.1^{\circ}$, 成功实现了对映体的区分. 2016 年, Thiele教授 ${ }^{[170]}$ 通过提高聚多肽的分子量, 增加液晶 


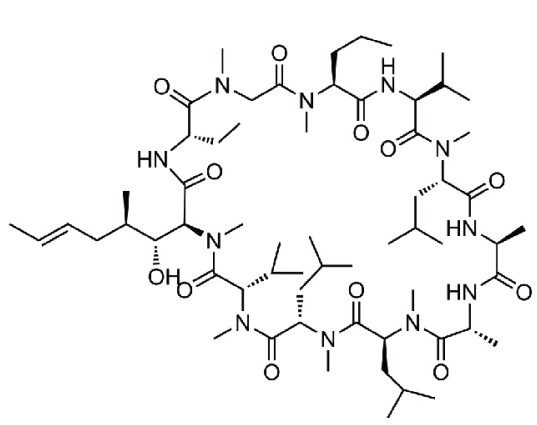

Cyclosporin A

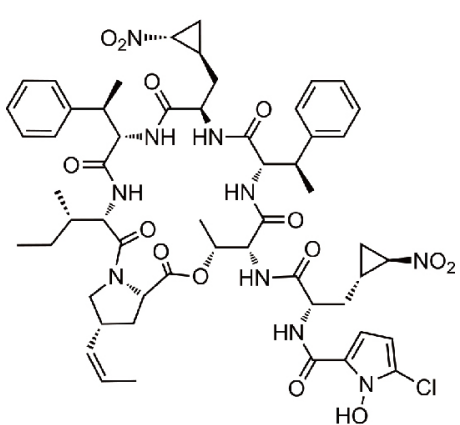

Hormaomycin

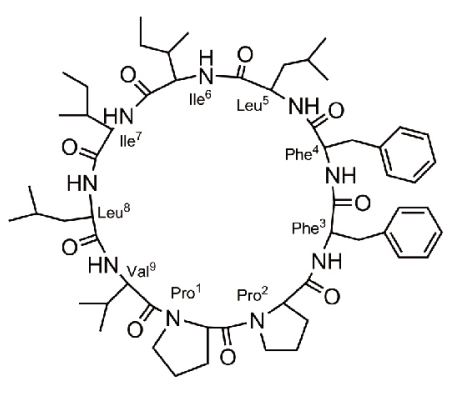

Cyclolinopeptide A

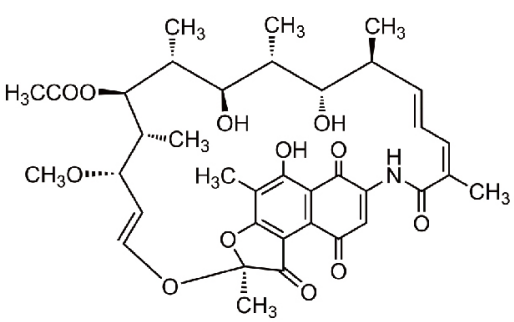

Rifamycin-S<smiles>CNC(=O)OC(CC(C)C)c1nc([C@H](OC(=O)C(C)=CCC(C)=CC(O)C(C)C=C(C)C)C(C)C)cs1</smiles><smiles>C/C=C\CCC(O)=C1C(=O)NC(C(O)CCNC(=O)/C=C\C=C/CC/C=C/C2CC3C(C)C=CC3(C)C2)C1=O</smiles><smiles>O=C1CC2C3N(CCC[C@]2(CI)CC(O)N3c2ccccc2)C1</smiles>

Archazolid A Cylindramide

Melohenine B

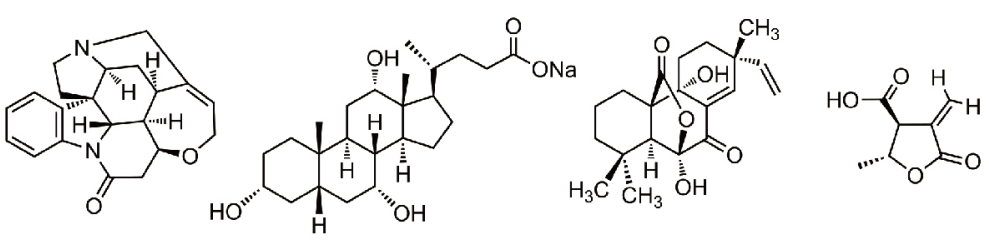

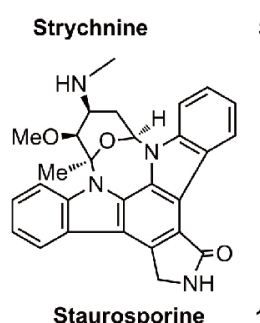
Sodium cholate Sphaeropsidin A Butyrolactone

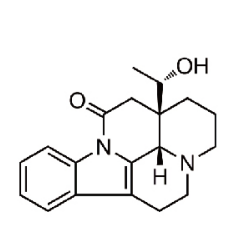<smiles>C=C1C(=O)O[C@H]2[C@H]1CCC(C)=C1C[C@H]3O[C@]3(C)[C@H]12</smiles><smiles>C=C1C(=O)O[C@H]2C1CC[C@@](C)(O)[C@@H]1CC=C(C)C21</smiles>

Staurosporin 19-OH-(-)-Eburnamonine Ludartin 10-epi-8-Deoxycumambrin B

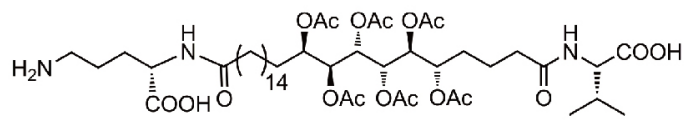

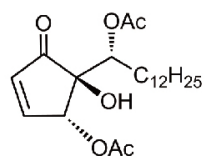

Sagittamide A

图 22 利用 RDC参数成功解析的天然产物结构 

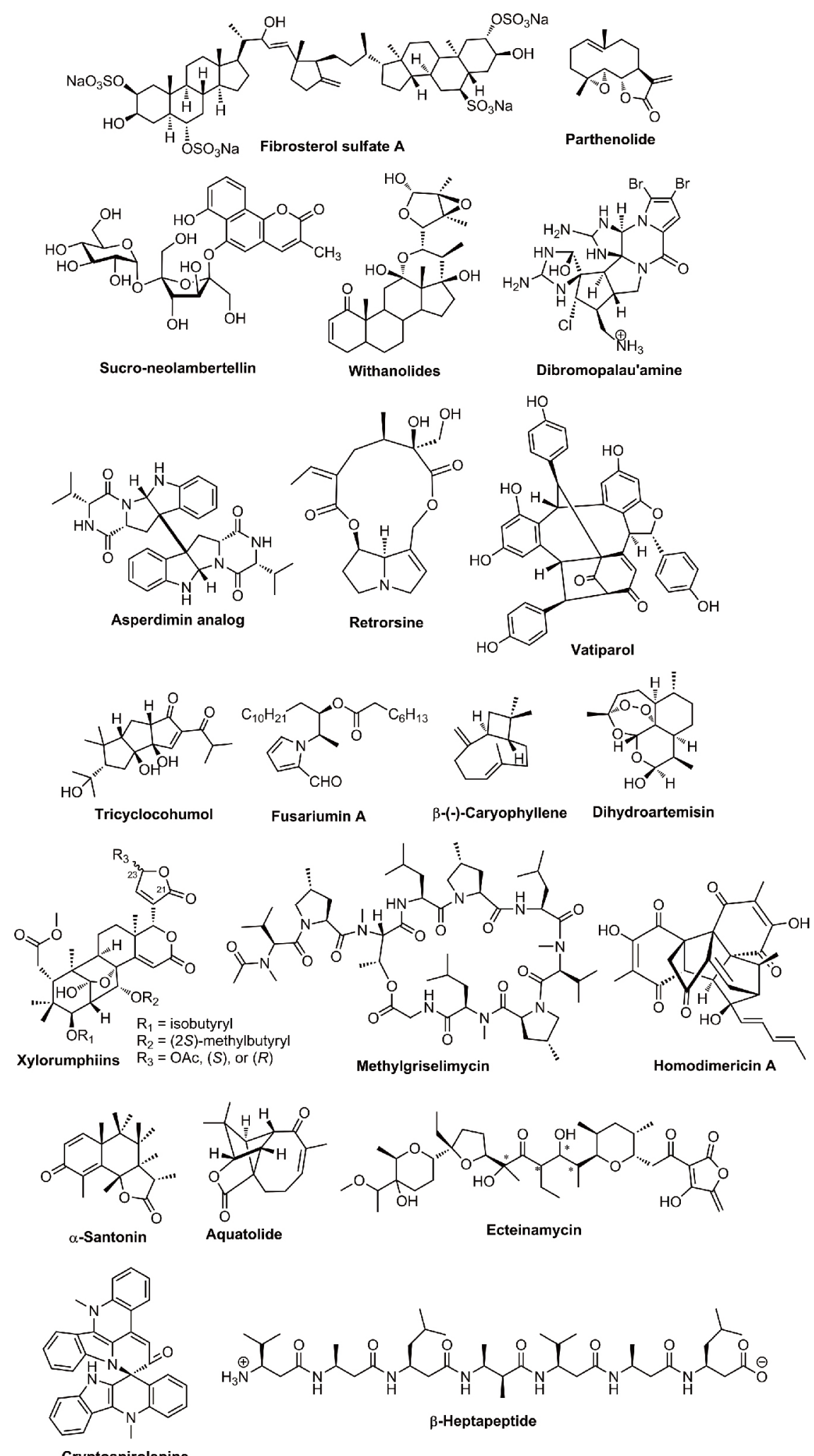
表 3 RDC在天然产物结构解析中的应用

\begin{tabular}{|c|c|c|c|c|}
\hline 天然产物 & 定向介质 & 年份 & 应用 & 文献 \\
\hline Strychnine & $\begin{array}{c}\mathrm{PBLG} / \mathrm{CDCl}_{3}, \\
\mathrm{PELG} / \mathrm{CDCl}_{3}, \mathrm{PS} / \mathrm{CDCl}_{3}\end{array}$ & $2003,2004,2005$ & prochiral assignment & {$[61,66,132,133]$} \\
\hline Sodium cholate & $\mathrm{PAA} / \mathrm{D}_{2} \mathrm{O}$ & 2003 & relative configuration & [25] \\
\hline Cyclosporin A & $\mathrm{PDMS} / \mathrm{CDCl}_{3}$ & 2005 & conformation & [125] \\
\hline Sphaeropsidin A & PVAc/DMSO & 2005 & prochiral assignment & [39] \\
\hline$\gamma$-Buty rolactone & $\left(\mathrm{C}_{12} \mathrm{E}_{5}\right) / n-\mathrm{Hexanol} / \mathrm{D}_{2} \mathrm{O}$ & 2006,2009 & relative configuration & {$[136 \sim 138]$} \\
\hline Hormaomycin & PH-PDMAA /DMSO & 2006 & conformation & [127] \\
\hline Sagittamide A & PH-PDMAA, PAN/DMSO & 2007 & relative configuration & [135] \\
\hline Ludartin & $\mathrm{PMMA} / \mathrm{CDCl}_{3}$ & 2008 & relative configuration & [33] \\
\hline Staurosporine & $\mathrm{dPS} / \mathrm{CDCl}_{3}$ & 2008 & relative configuration, & [30] \\
\hline Cylindramide & PAN/DMSO & 2008 & conformation & [128] \\
\hline Archazolide A & PH-PDMAA/DMSO & 2008 & $\begin{array}{l}\text { relative configuration, } \\
\text { conformation }\end{array}$ & [129] \\
\hline Sucro-neolambertellin & PH-PDMAA, PAN/DMSO & 2008 & relative configuration & [141] \\
\hline Withanolides & $\mathrm{PMMA} / \mathrm{CDCl}_{3}$ & 2009 & absolute configuration & [134] \\
\hline Tricyclocohumol & dPAN/DMSO & 2009 & relative configuration & [145] \\
\hline 10-epi-8-Deoxycumambrin B & $\mathrm{PMMA} / \mathrm{CDCl}_{3}$ & 2010 & relative configuration & {$[34,114]$} \\
\hline (-)-Dibromopalau'amine & PAN/DMSO & 2010 & absolute configuration & [142] \\
\hline Parthenolide & PAN/DMSO & 2010 & conformation & [29] \\
\hline Fibrosterol sulfate A & dPAN/DMSO & 2011 & relative configuration & [140] \\
\hline Asperdimin analog & $\mathrm{PDMS} / \mathrm{CDCl}_{3}$ & 2011 & $\begin{array}{l}\text { relative and absolute } \\
\text { configuration }\end{array}$ & [144] \\
\hline Vatiparol & $\mathrm{PH} / \mathrm{DMSO}$ & 2012 & absolute configuration & [146] \\
\hline 19-OH-(-)-Eburnamonine & $\mathrm{PMMA} / \mathrm{CDCl}_{3}$ & 2012 & absolute configuration & [143] \\
\hline 4,6-Diacetylhygrophorone $\mathrm{A}^{12}$ & $\mathrm{PBLG} / \mathrm{CD}_{2} \mathrm{Cl}_{2}$ & 2013 & relative configuration & [139] \\
\hline Rifamycin-S & $\mathrm{PDMS} / \mathrm{CDCl}_{3}$ & 2013 & $\begin{array}{l}\text { relative configuration, } \\
\text { conformation }\end{array}$ & [126] \\
\hline Cyclolinopeptide A & $\mathrm{PDMS} / \mathrm{CDCl}_{3}$ & 2014 & conformation & [130] \\
\hline Melohenine B & $\mathrm{PBLG} / \mathrm{CDCl}_{3}$ & 2015 & conformation & [131] \\
\hline$\alpha$-Santonin & PAN/DMSO & 2015 & relative configuration & [147] \\
\hline Retrorsine & p-HEMA, PMMA/DMSO & 2016 & conformation & {$[43,16]$} \\
\hline Fusariumin A & $\mathrm{PH} / \mathrm{DMSO}$ & 2016 & absolute configuration & [148] \\
\hline Dihydroartemisin & GO-g-TFEMA/DMSO & 2016 & configuration & [84] \\
\hline Homodimericin A & p-HEMA/DMSO & 2016 & relative configuration & [149] \\
\hline$\beta-(-)$-Caryophyllene & $\mathrm{PDMS} / \mathrm{CDCl}_{3}$ & 2017 & conformation & [32] \\
\hline Xylorumphiins & $\mathrm{PMMA} / \mathrm{CDCl}_{3}$ & 2017 & relative configuration & [150] \\
\hline Methylgriselimycin & $\mathrm{PDMS} / \mathrm{CDCl}_{3}$ & 2017 & conformation & [151] \\
\hline Cryptospirolepine & p-HEMA/DMSO & 2017 & relative configuration & [152] \\
\hline Aquatolide & p-HEMA/DMSO & 2017 & relative configuration & [152] \\
\hline Ecteinamycin & $\mathrm{PMMA} / \mathrm{CDCl}_{3}$ & 2017 & relative configuration & [153] \\
\hline$\beta$-Heptapeptide & $\mathrm{PVAc} / \mathrm{CD}_{3} \mathrm{OH}$ & 2017 & relative configuration & [154] \\
\hline
\end{tabular}



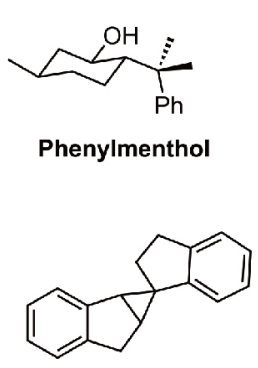

Spiroindene

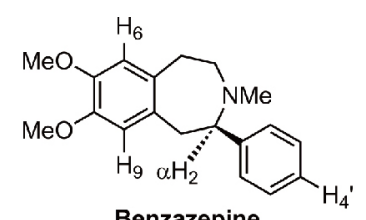

Benzazepine

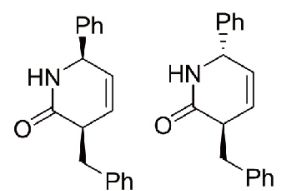

Dihydropyridone (cis/trans)<smiles>COc1c(C)c2c(c(C)c1OC)[C@@H](C)[C@H](C)N[C@H](C)[C@H]2C</smiles>

Salsolidine $\mathrm{HCl}$

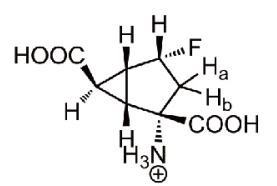

Bicyclic glutamate analog

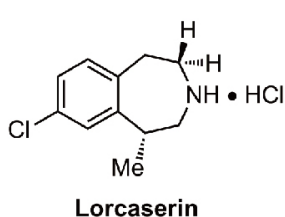<smiles>CC(C)(C)c1cccc2c([C@@H](O)[C@@H]3CCCCN3)cc(C(F)(F)F)nc12</smiles><smiles>CO[R9]O[Si]</smiles><smiles>CC12CC(c3ccccc3)=C(I)CC13CCCN23</smiles>

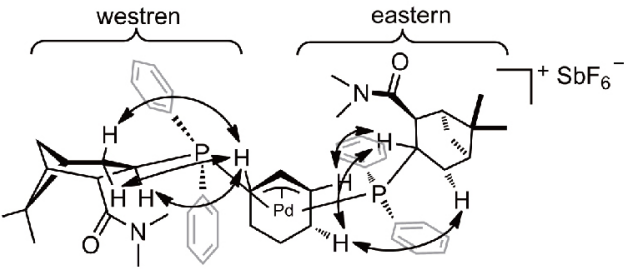

Pd-morphous complex

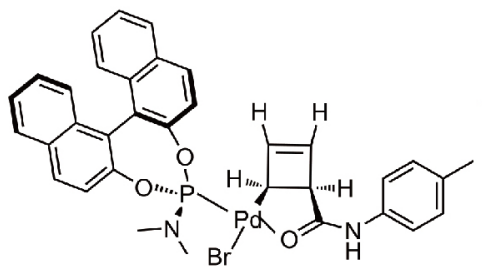

Pd-complexes A

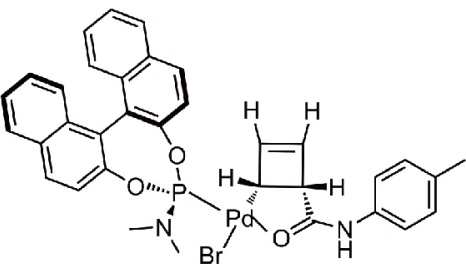

Pd-complexes B<smiles>CC1(C)O[C@H]2CO[C@]3(CC[C@]4(OC[C@H]5OC(C)(C)O[C@H]5C4=O)O3)[C@H](O)[C@@H]2O1</smiles><smiles>COc1ccc2c(c1)[C@@]13CCCC[C@H]1[C@H](C2)N(C)C3</smiles>

Dextromethorphan

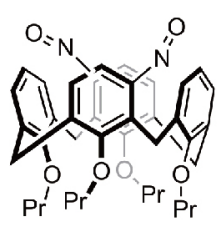<smiles>C[C@H](C(=O)N1C(=O)OC[C@H]1Br)[C@H](CC(=O)c1ccccc1)c1ccc2c(c1)OCO2</smiles>

Calix[4]arene

Michael adduct

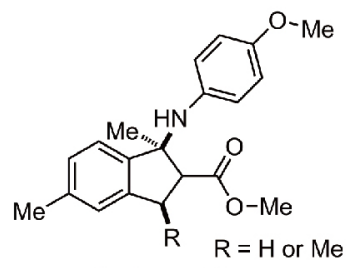

B-Amino acid esters

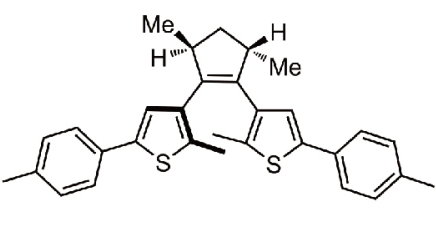

Dithienylcyclopentene

图 23 利用 RDC 参数成功解析的合成药物、有机反应中间体和反应产物的结构 
表 4 RDC在合成药物或有机中间体结构分析中的应用

\begin{tabular}{|c|c|c|c|c|}
\hline 合成分子 & 定向介质 & 年份 & 应用 & 文献 \\
\hline Dihydropyridone (cis/trans) & PBLG-PBDG mix/ $\mathrm{CDCl}_{3}$ & 2003 & relative configuration & [63] \\
\hline Spiroindene & $\mathrm{PDMS} / \mathrm{CDCl}_{3}$ & 2004 & relative configuration & {$[1,31]$} \\
\hline Bicyclic glutamate analog & pf1 phage-bicelles $/ \mathrm{D}_{2} \mathrm{O}$ & 2004 & relative configuration & [155] \\
\hline Phenylmenthol & $\mathrm{PELG} / \mathrm{CDCl}_{3}$ & 2009 & configuration & {$[156]$} \\
\hline Benzazepine & $\mathrm{PDMS} / \mathrm{CD}_{2} \mathrm{Cl}_{2}$ & 2010 & conformation & [157] \\
\hline Pd-morphous complex & PELG/CD $\mathrm{Cl}_{2}$ & 2010 & conformation & [158] \\
\hline Salsolidine $\cdot \mathrm{HCl}$ & $\mathrm{CPCL} / \mathrm{D}_{2} \mathrm{O}$ & 2010 & conformation & [159] \\
\hline Dextromethorphan & $\mathrm{PS} / \mathrm{CDCl}_{3}$ & 2010 & relative configuration & {$[160]$} \\
\hline Tricyclic aziridine & $\mathrm{PS} / \mathrm{CDCl}_{3}$ & 2011 & constitution, configuration & {$[17,161]$} \\
\hline Michael adduct & $\mathrm{PAN} / / \mathrm{DMSO}$ & 2011 & relative/absolute configuration & [162] \\
\hline Mefloquine $\cdot \mathrm{HCl}$ & $\begin{array}{c}(S)-\mathrm{APhES}- \\
\text { PDMAA/CD } \\
\text { OD }\end{array}$ & 2012 & absolute configuration & [163] \\
\hline Lorcaserin & $\begin{array}{l}\text { AMPS-acrylamide } \\
\text { copolymer } / \mathrm{D}_{2} \mathrm{O}\end{array}$ & 2013 & conformation & {$[164]$} \\
\hline Calix[4]arene & $\begin{array}{l}\text { PBLG or polyacetylene/ } \\
\qquad \mathrm{CDCl}_{3}\end{array}$ & 2014 & conformation & {$[165]$} \\
\hline Pd-complexesAandB & PDMS/THF- $d_{8}$ & 2015,2017 & constitution, conformation & {$[32,166]$} \\
\hline Dithienylcyclopentene & PBLG gel $/ \mathrm{CDCl}_{3}$ & 2015 & conformation & [167] \\
\hline Spiroepoxide & $\mathrm{PELG} / \mathrm{CDCl}_{3}$ & 2015 & constitution, relative configuration & {$[168]$} \\
\hline$\beta$-Amino acid esters & $\mathrm{PS} / \mathrm{CDCl}_{3}$ & 2015 & relative configuration & [169] \\
\hline Spiroketal & p-HEMA/DMSO & 2017 & relative configuration, conformation & [152] \\
\hline
\end{tabular}

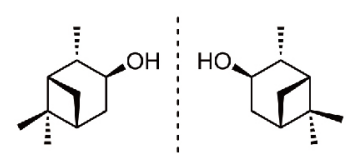

(+)-IPC

$(-)-I P C$<smiles>CC1CC[C@@H](C(C)C)[C@H](N)C1</smiles>

(+)-Menthylamine $\mathrm{HCl}$<smiles>CC(C)C1CC[C@@H](C)C[C@H]1NCCl</smiles>

(+)-Menthylamine $\mathrm{HCl}$
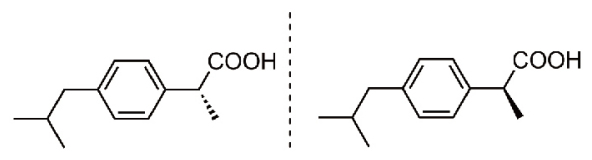

$(R)$ - and (S)-ibuprofen<smiles>C=C1CC[C@@H]2CC1[C@]2(C)I</smiles>

(+)- $\beta$-pinene

$(-)-\beta$-pinene<smiles>CC1CCC(C(C)C)C(O)C1</smiles>

(+)-Menthol<smiles>CC1CCC(C(C)C)C(O)C1</smiles>

(-)-Menthol
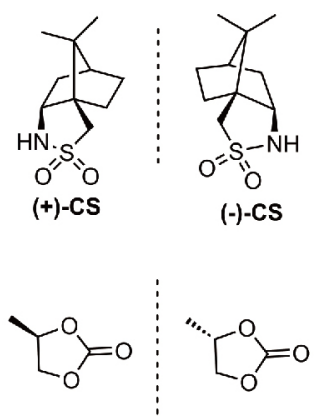

$(R)$ - and (S)-propylene carbonate
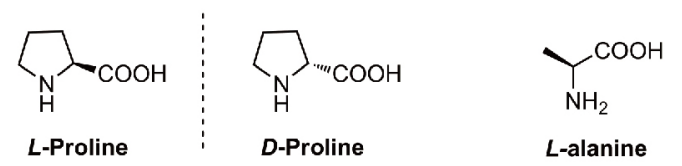

$$
\text { ".. } \mathrm{NH}_{2} \mathrm{COOH}
$$

D-alanine

图 $24 \mathrm{RDC}$ 手性区分对映体代表性的有机分子结构 
自组装长度, 合成了更高分子量的PBLG和PELG, 并比 较了它们识别对映体IPC和蒎烯 $\beta$-pinene的能力: PELG 液晶的识别性能要优于PBLG液晶. 2017年Thiele教授 等 ${ }^{[171]}$ 又通过修饰PBLG或PELG的末端酯基结构, 引入 (S)-手性异戊基辅基边链形成的手性液晶PSMBLG和 PSMBDG (图25), 它们对(+)-IPC和(-)-IPC的手性识别 $\beta$ 角度分别是 $16.6^{\circ}$ 和 $23.4^{\circ}$, 同时在PSMBDG 液晶中也 研究了天然产物(-)-curcumol的结构. 同年, Thiele教授 等 ${ }^{[172]}$ 又发展了一种温控性手性聚谷氨酸苯乙酯PPLA 和PPDA的四氯乙烷液晶, 对(+)-IPC和(-)-IPC的手性 识别 $\beta$ 角度分别达到 $43.4^{\circ}$ 和 $44.3^{\circ}$, 当液晶温度提高至 $120^{\circ} \mathrm{C}$ 时, 其识别效果仍然可观 $\left(\beta=19.1^{\circ}, 19.8^{\circ}\right)$ (图25).

Kuchel等 ${ }^{[173]}$ 报道了拉伸或压缩聚多糖类的手性 胶, 即Iota-和Kappa-carageenan定向介质(图26). Kappa-<smiles>CCC(C)COC(=O)CCC(NC)C(C)=O</smiles>

PSMBLG<smiles>CNC(CC(=O)OCCc1ccccc1)C(C)=O</smiles>

PPLA

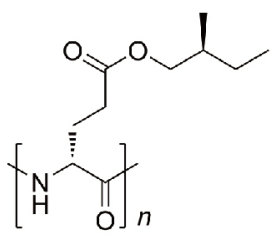

PSMBDG

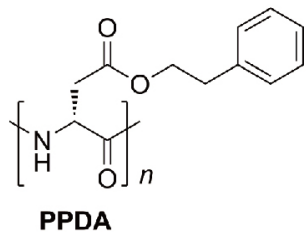

图 25 手性PSMBLG及PSMBDG 与PPLA及PPDA液晶定 向介质的结构 ${ }^{[171,172]}$
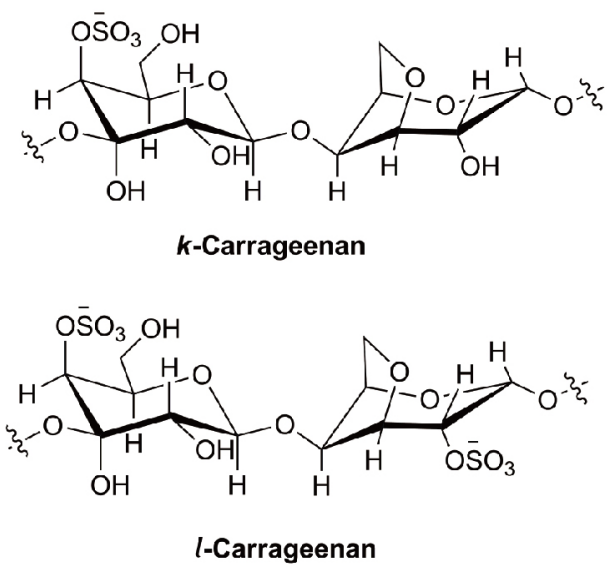

图 26 聚多糖手性胶 Iota-和kappa-carrageenan结构 ${ }^{[173]}$ carageenan胶在水溶液中能区分丙氨酸消旋体和确定 甘氨酸的潜手性H原子, 而Iota-carageenan介质能观察 到DMSO- $d_{6}$ 溶剂中 $\mathrm{CD}_{3}$ 的氛裂分, 其性质与Luy等报道 的明胶gelatin手性介质类似. 将缅氨酸的对映异构体按 照 $L$-烦氨酸 $/ D$-顺氨酸 $=1.2: 1$ 的比例扩散进手性明胶内, 成功实现了对映异构体的区分 ${ }^{\left[{ }^{[7]}\right.}$. 而Reinscheid等 ${ }^{[38]}$ 以 聚丙烯酰胺为基础发展了 $(R)$-APhES或 $(S)$-APhES-PH 胶手性介质(见2.1小节, 图6), 能溶解于水和有机溶剂 二甲亚砜、甲醇中, 可实现对有机胺类的对映体区分. 能有效区分甲氟喹盐酸盐 $\left(\beta=11.3^{\circ}\right)$ 、马钱子碱盐酸盐 $\left(\beta=16.8^{\circ}\right)$ 、薄荷胺盐酸盐 $\left(\beta=40.5^{\circ}\right)$ 的对映异构体, 其 机理可能是定向介质中含有酸性基团与待测化合物 的氨基发生了弱的相互作用.

2007 年, 通过相关RDC参数研究了 $(R)$ 或 $(S)$-布洛 芬(ibuprofen)异构体在PBLG/CDCl 3 液晶中的不同, 这 种方法对拥有一个或两个手性中心, 可溶于低黏度有 机溶剂并且不会结晶析出的有机小分子也适用 ${ }^{[174]}$. 而 Suryaprakash教授等 ${ }^{[175,176]}$ 利用二维C-HETSERF, INADEQUATE实验在 $P B L G / \mathrm{CDCl}_{3}$ 手性液晶中, 提取同核或 杂核 ${ }^{1} D_{\mathrm{C}-\mathrm{H}},{ }^{1} D_{\mathrm{C}-\mathrm{C}}$ 和 ${ }^{1} D_{\mathrm{H}-\mathrm{H}}$, 经过DFT模型计算, 清晰地确 认了丙烯酸碳酸酯对映体.

\section{7 总结与展望}

利用残留偶极耦合构建分子的空间结构是核磁 共振波谱学、生物大分子结构、有机分析研究领域 的前沿和热点. RDC参数的测定方法及应用RDC解析 相关分子结构的研究工作目前已呈现蓬勃发展之势. 而应用残留偶极耦合的先决条件是弱定向介质的构 建, 因此随着性能优异的定向介质被不断开发, 更多 结构复杂多变的有机分子的鉴定瓶颈问题被逐渐破 除. 为实现这种方法在实验室的常规检测, 更优秀的 手性定向介质开发是未来发展的一个重要方向, 为解 决许多新结构的绝对构型或对映体过量分析的测定 提供更多选择. 同时随着定向介质中检测脉冲技术不 断优化与修正, 为解决远程残留各向异性参数的测定 方面提供可能. 而柔性分子构象分布的计算目前仍具 挑战, 限制了当前RDC主要用于刚性和半刚性分子, 或柔性大分子中的较大刚性片段的分析. 但计算机辅 助的分子模拟构型和构象分析软件的发展, 为柔性有 机分子的结构模型的建立提供便利. 更有想象空间的 是随着人工智能的发展, 采用 $\mathrm{RDC}$ 全局约束的特征, 
可能被用于有机分子结构的图谱自动归属及结构鉴 定. 在若干年内, RDC可能成为实验室常用的结构鉴
定和推断方法, 实现RDC技术在有机化学领域的广泛 应用。

\section{参考文献}

1 Yan J, Zartler ER. Magn Reson Chem, 2005, 43: 53-64

2 Gschwind RM. Angew Chem Int Ed, 2005, 44: 4666-4668

3 Luy B, Kessler H. Modern Magn Reson, 2006, 1279-1285

4 Thiele CM. Eur J Org Chem, 2008, 2008: 5673-5685

5 Annila A, Permi P. Concepts Magn Reson, 2004, 23A: 22-37

6 Thiele CM. Concepts Magn Reson, 2007, 30A: 65-80

7 Kummerlöwe G, Luy B. TrAC Trends Anal Chem, 2009, 28: 483-493

8 Kummerlöwe G, Luy B. Annu Rep NMR Spectrosc, 2009, 68: 193-232

9 Böttcher B, Thiele CM. eMagRes, 2012, 1: 169-180

10 Canales A, Jiménez-Barbero J, Martín-Pastor M. Magn Reson Chem, 2012, 50: S80-S85

11 Batista Jr JM, Blanch EW, Bolzani VS. Nat Prod Rep, 2015, 32: 1280-1302

12 Schmidts V. Magn Reson Chem, 2017, 55: 54-60

13 Gil RR, Griesinger C, Navarro-Vázquez A, Sun H. Structural elucidation of small organic molecules assisted by NMR in aligned media. In: Cid MM, Bravo J, Eds. Structure Elucidation in Organic Chemistry: the Search for the Right Tools: 1st Ed. Weinheim: Wiley-VCH, 2014. 279-323

14 Gil RR, Navarro-Vázquez A. Application of residual dipolar couplings to the structural analysis of natural products. In: Williams A, Martin G, Rovnyak D, Eds. Modern NMR Approaches to the Structure Elucidation of Natural Products. Vol. 2, Chapter 4. Cambridge, UK: The Royal Society of Chemistry, 2016. 117-176

15 Hellemann E, Teles RR, Hallwass F, Barros Jr W, Navarro-Vázquez A, Gil RR. Chem Eur J, 2016, 22: 16632-16635

16 Nath N, Schmidt M, Gil RR, Williamson RT, Martin GE, Navarro-Vázquez A, Griesinger C, Liu Y. J Am Chem Soc, 2016, 138: 9548-9556

17 Gil RR. Angew Chem Int Ed, 2011, 50: 7222-7224

18 Kummerlöwe G, Grage SL, Thiele CM, Kuprov I, Ulrich AS, Luy B. J Magn Reson, 2011, 209: 19-30

19 Hallwass F, Schmidt M, Sun H, Mazur A, Kummerlöwe G, Luy B, Navarro-Vázquez A, Griesinger C, Reinscheid UM. Angew Chem Int Ed, 2011, 50: 9487-9490

20 Wirz LN, Allison JR. J Biomol NMR, 2015, 62: 25-29

21 Saupe A, Englert G. Phys Rev Lett, 1963, 11: 462-464

22 Saupe A. Z für Naturforschung A, 1964, 19: 161-171

23 Tjandra N, Bax A. Science, 1997, 278: 1111-1114

24 Yan J, Kline AD, Mo H, Shapiro MJ, Zartler ER. J Org Chem, 2003, 68: 1786-1795

25 Mangoni A, Esposito V, Randazzo A. Chem Commun, 2003, 154-155

26 Prestegard JH, Bougault CM, Kishore AI. Chem Rev, 2004, 104: 3519-3540

27 Luy B, Kobzar K, Kessler H. Angew Chem Int Ed, 2004, 43: 1092-1094

28 Kummerlöwe G, Halbach F, Laufer B, Luy B. Open Spectrosc J, 2008, 2: 29-33

29 Kummerlöwe G, McCord EF, Cheatham SF, Niss S, Schnell RW, Luy B. Chem Eur J, 2010, 16: 7087-7089

30 Kummerlöwe G, Knör S, Frank AO, Paululat T, Kessler H, Luy B. Chem Commun, 2008, 12: 5722

31 Freudenberger JC, Spiteller P, Bauer R, Kessler H, Luy B. J Am Chem Soc, 2004, 126: 14690-14691

32 Moskalenko YE, Bagutski V, Thiele CM. Chem Commun, 2017, 53: 95-98

33 Gil RR, Gayathri C, Tsarevsky NV, Matyjaszewski K. J Org Chem, 2008, 73: 840-848

34 Gayathri C, Tsarevsky NV, Gil RR. Chem Eur J, 2010, 16: 3622-3626

35 Specialty NMR sample tubes, compression gel device. Vineland, NJ: New Era Enterprises, Inc. http://www.newera-spectro.com

36 Montag T, Thiele CM. Chem Eur J, 2013, 19: 2271-2274

37 Haberz P, Farjon J, Griesinger C. Angew Chem Int Ed, 2005, 44: 427-429

38 Schmidt M, Sun H, Leonov A, Griesinger C, Reinscheid UM. Magn Reson Chem, 2012, 50: S38-S44

39 Freudenberger JC, Knör S, Kobzar K, Heckmann D, Paululat T, Kessler H, Luy B. Angew Chem Int Ed, 2005, 44: 423-426 
Kummerlöwe G, Auernheimer J, Lendlein A, Luy B. J Am Chem Soc, 2007, 129: 6080-6081

Kummerlöwe G, Behl M, Lendlein A, Luy B. Chem Commun, 2009, 46: 8273

Merle C, Kummerlöwe G, Freudenberger JC, Halbach F, Stöwer W, Gostomski CL, Höpfner J, Beskers T, Wilhelm M, Luy B. Angew Chem Int Ed, 2013, 52: 10309-10312

Gil-Silva LF, Santamaría-Fernández R, Navarro-Vázquez A, Gil RR. Chem Eur J, 2016, 22: 472-476

García ME, Woodruff SR, Hellemann E, Tsarevsky NV, Gil RR. Magn Reson Chem, 2017, 55: 206-209

Tycko R, Blanco FJ, Ishii Y. J Am Chem Soc, 2000, 122: 9340-9341

Sass HJ. J Biomol NMR, 2000, 18: 303-309

Kobzar K, Kessler H, Luy B. Angew Chem Int Ed, 2005, 44: 3145-3147

Kummerlöwe G, Kiran MU, Luy B. Chem Eur J, 2009, 15: 12192-12195

Eliav U, Navon G. J Am Chem Soc, 2006, 128: 15956-15957

Ma J, Goldberg GI, Tjandra N. J Am Chem Soc, 2008, 130: 16148-16149

Broer DJ, Bastiaansen CMW, Debije MG, Schenning APHJ. Angew Chem Int Ed, 2012, 51: 7102-7109

Giese M, Blusch LK, Khan MK, MacLachlan MJ. Angew Chem Int Ed, 2015, 54: 2888-2910

Doty P, Holtzer AM, Bradbury JH, Blout ER. J Am Chem Soc, 1954, 76: 4493-4494

Meddour A, Canet I, Loewenstein A, Pechine JM, Courtieu J. J Am Chem Soc, 1994, 116: 9652-9656

Canet I, Meddour A, Courtieu J, Canet JL, Salaun J. J Am Chem Soc, 1994, 116: 2155-2156

Canet I, Courtieu J, Loewenstein A, Meddour A, Pechine JM. J Am Chem Soc, 1995, 117: 6520-6526

Lesot P, Merlet D, Loewenstein A, Courtieu J. Tetrahedron-Asymmetry, 1998, 9: 1871-1881

Merlet D, Ancian B, Courtieu J, Lesot P. J Am Chem Soc, 1999, 121: 5249-5258

Sarfati M, Lesot P, Merlet D, Courtieu J. Chem Commun, 2000: 2069-2081

Canlet C, Merlet D, Lesot P, Meddour A, Loewenstein A, Courtieu J. Tetrahedron-Asymmetry, 2000, 11: 1911-1918

Thiele CM, Berger S. Org Lett, 2003, 5: 705-708

Verdier L, Sakhaii P, Zweckstetter M, Griesinger C. J Magn Reson, 2003, 163: 353-359

Aroulanda C, Boucard V, Guibé F, Courtieu J, Merlet D. Chem Eur J, 2003, 9: 4536-4539

Aroulanda C, Sarfati M, Courtieu J. Enantiomer, 2001, 6: 281-287

Lesot P, Sarfati M, Courtieu J. Chem Eur J, 2003, 9: 1724-1745

Thiele CM. J Org Chem, 2004, 69: 7403-7413

Marx A, Schmidts V, Thiele CM. Magn Reson Chem, 2009, 47: 734-740

Marx A, Böttcher B, Thiele CM. Chem Eur J, 2010, 16: 1656-1663

Luy B. J Indina Inst Sci, 2012, 90: 119-132

Marx A, Thiele C. Chem Eur J, 2009, 15: 254-260

Thiele CM, Pomerantz WC, Abbott NL, Gellman SH. Chem Commun, 2011, 47: 502-504

Lei X, Qiu F, Sun H, Bai L, Wang WX, Xiang W, Xiao H. Angew Chem Int Ed, 2017, 56: 12857-12861

Arnold L, Marx A, Thiele CM, Reggelin M. Chem Eur J, 2010, 16: 10342-10346

Meyer NC, Krupp A, Schmidts V, Thiele CM, Reggelin M. Angew Chem Int Ed, 2012, 51: 8334-8338

Krupp A, Reggelin M. Magn Reson Chem, 2012, 50: S45-S52

Dama M, Berger S. Tetrahedron Lett, 2012, 53: 6439-6442

Dama M, Berger S. Org Lett, 2012, 14: 241-243

Reller M, Wesp S, Koos MRM, Reggelin M, Luy B. Chem Eur J, 2017, 23: 13351-13359

刘亚婷. 新型残留偶极耦合提取介质的合成与应用. 硕士学位论文. 温州: 温州大学, 2015

李高伟. 残留偶极耦合参数在有机分子结构解析及NMR波谱在手性识别中的应用. 博士学位论文. 南京: 南京大学, 2017

Li GW, Cao JM, Zong W, Hu L, Hu ML, Lei X, Sun H, Tan RX. Chem Eur J, 2017, 23: 7653-7656

Leyendecker M, Meyer NC, Thiele CM. Angew Chem Int Ed, 2017, 56: 11471-11474

Lei X, Xu Z, Sun H, Wang S, Griesinger C, Peng L, Gao C, Tan RX. J Am Chem Soc, 2014, 136: 11280-11283

Zong W, Li GW, Cao JM, Lei X, Hu ML, Sun H, Griesinger C, Tan RX. Angew Chem Int Ed, 2016, 55: 3690-3693

França JAA, Navarro-Vázquez A, Lei X, Sun H, Griesinger C, Hallwass F. Magn Reson Chem, 2017, 55: 297-303

Hansen MR, Mueller L, Pardi A. Nat Struct Mol Biol, 1998, 5: 1065-1074

Rückert M, Otting G. J Am Chem Soc, 2000, 122: 7793-7797

Klochkov VV, Klochkov AV, Thiele CM, Berger S. J Magn Reson, 2006, 179: 58-63 
Pham TN, Hinchley SL, Rankin DWH, Liptaj T, Uhrín D. J Am Chem Soc, 2004, 126: 13100-13110

Dama M, Berger S. Carbohyd Res, 2013, 377: 44-47

Di Pietro ME, Celebre G, De Luca G. J Magn Reson, 2016, 267: 63-67

Troche-Pesqueira E, Cid MM, Navarro-Vázquez A. Org Biomol Chem, 2014, 12: 1957-1965

Lokesh , Suryaprakash N. Chem Commun, 2013, 49: 2049

Enthart A, Freudenberger JC, Furrer J, Kessler H, Luy B. J Magn Reson, 2008, 192: 314-322

Kaltschnee L, Kolmer A, Timári I, Schmidts V, Adams RW, Nilsson M, Kövér KE, Morris GA, Thiele CM. Chem Commun, 2014, 50: $15702-15705$

Timári I, Kaltschnee L, Kolmer A, Adams RW, Nilsson M, Thiele CM, Morris GA, Kövér KE. J Magn Reson, 2014, 239: 130-138

Zangger K. Prog Nucl Magn Reson Spectr, 2015, 86-87: 1-20

Becker J, Luy B. Magn Reson Chem, 2015, 53: 878-885

Wu Z, Bax A. J Magn Reson, 2001, 151: 242-252

Tian F, Bolon PJ, Prestegard JH. J Am Chem Soc, 1999, 121: 7712-7713

Yu B, van Ingen H, Freedberg DI. J Magn Reson, 2013, 228: 159-165

Schwalbe H, Marino JP, King GC, Wechselberger R, Bermel W, Griesinger C. J Biomol NMR, 1994, 4: 631-644

Martín-Pastor M, Canales-Mayordomo A, Jiménez-Barbero J. J Biomol NMR, 2003, 26: 345-353

Carlomagno T, Peti W, Griesinger C. J Biomol NMR, 2000, 17: 99-109

Tzvetkova P, Simova S, Luy B. J Magn Reson, 2007, 186: 193-200

Jin L, Uhrín D. Magn Reson Chem, 2007, 45: 628-633

Parella T, Espinosa JF. Prog Nucl Magn Reson Spectr, 2013, 73: 17-55

Nath N, d'Auvergne EJ, Griesinger C. Angew Chem Int Ed, 2015, 54: 12706-12710

Uhrín D, Batta G, Hruby VJ, Barlow PN, Kövér KE. J Magn Reson, 1998, 130: 155-161

Meissner A, Sørensen OW. Magn Reson Chem, 2001, 39: 49-52

Bax A, Freeman R, Kempsell SP. J Am Chem Soc, 1980, 102: 4849-4851

Furrer J, John M, Kessler H, Luy B. J Biomol NMR, 2007, 37: 231-243

Thiele CM, Bermel W. J Magn Reson, 2012, 216: 134-143

Trigo-Mouriño P, Navarro-Vázquez A, Ying J, Gil RR, Bax A. Angew Chem Int Ed, 2011, 50: 7576-7580

Snider JD, Troche-Pesqueira E, Woodruff SR, Gayathri C, Tsarevsky NV, Gil RR. Magn Reson Chem, 2012, 50: S86-S91

Castañar L, Garcia M, Hellemann E, Nolis P, Gil RR, Parella T. J Org Chem, 2016, 81: 11126-11131

Zweckstetter M. Nat Protoc, 2008, 3: 679-690

Navarro-Vázquez A. Magn Reson Chem, 2012, 50: S73-S79

Berger R, Fischer C, Klessinger M. J Phys Chem A, 1998, 102: 7157-7167

Cornilescu G, Marquardt JL, Ottiger M, Bax A. J Am Chem Soc, 1998, 120: 6836-6837

Losonczi JA, Andrec M, Fischer MWF, Prestegard JH. J Magn Reson, 1999, 138: 334-342

Kramer F, Deshmukh MV, Kessler H, Glaser SJ. Concepts Magn Reson, 2004, 21A: 10-21

Sass J, Cordier F, Hoffmann A, Rogowski M, Cousin A, Omichinski JG, Löwen H, Grzesiek S. J Am Chem Soc, 1999, 121: 2047-2055

Berger R, Courtieu J, Gil RR, Griesinger C, Köck M, Lesot P, Luy B, Merlet D, Navarro-Vázquez A, Reggelin M, Reinscheid UM, Thiele CM, Zweckstetter M. Angew Chem Int Ed, 2012, 51: 8388-8391

Klages J, Neubauer C, Coles M, Kessler H, Luy B. ChemBioChem, 2005, 6: 1672-1678

Kakita VMR, Rachineni K, Bharatam J. J Mol Struct, 2013, 1053: 122-126

Reinscheid UM, Farjon J, Radzom M, Haberz P, Zeeck A, Blackledge M, Griesinger C. ChemBioChem, 2006, 7: 287-296

Cramer N, Helbig S, Baro A, Laschat S, Diestel R, Sasse F, Mathieu D, Richter C, Kummerlöwe G, Luy B, Schwalbe H. ChemBioChem, 2008, 9: $2474-2486$

Farès C, Hassfeld J, Menche D, Carlomagno T. Angew Chem Int Ed, 2008, 47: 3722-3726

Huben K, Jewgiński M, Pabis A, Paluch P, Luy B, Jankowski S. J Pept Sci, 2014, 20: 901-907

Lancefield CS, Slawin AMZ, Westwood NJ, Lebl T. Magn Reson Chem, 2015, 53: 467-475

Luy B, Kobzar K, Knör S, Furrer J, Heckmann D, Kessler H. J Am Chem Soc, 2005, 127: 6459-6465

Thiele CM. Angew Chem Int Ed, 2005, 44: 2787-2790

García ME, Pagola S, Navarro-Vázquez A, Phillips DD, Gayathri C, Krakauer H, Stephens PW, Nicotra VE, Gil RR. Angew Chem Int Ed, 2009, 48: $5670-5674$ 
Schuetz A, Junker J, Leonov A, Lange OF, Molinski TF, Griesinger C. J Am Chem Soc, 2007, 129: 15114-15115

Thiele CM, Marx A, Berger R, Fischer J, Biel M, Giannis A. Angew Chem Int Ed, 2006, 45: 4455-4460

Thiele CM, Maliniak A, Stevensson B. J Am Chem Soc, 2009, 131: 12878-12879

Thiele CM, Schmidts V, Böttcher B, Louzao I, Berger R, Maliniak A, Stevensson B. Angew Chem Int Ed, 2009, 48: 6708-6712

Schmidts V, Fredersdorf M, Lübken T, Porzel A, Arnold N, Wessjohann L, Thiele CM. J Nat Prod, 2013, 76: 839-844

Sun H, Reinscheid UM, Whitson EL, d'Auvergne EJ, Ireland CM, Navarro-Vázquez A, Griesinger C. J Am Chem Soc, 2011, 133: 14629-14636

Schuetz A, Murakami T, Takada N, Junker J, Hashimoto M, Griesinger C. Angew Chem Int Ed, 2008, 47: 2032-2034

Reinscheid UM, Köck M, Cychon C, Schmidts V, Thiele CM, Griesinger C. Eur J Org Chem, 2010, 2010: 6900-6903

Trigo-Mouriño P, Sifuentes R, Navarro-Vázquez A, Gayathri C, Maruenda H, Gil RR. Nat Prod Commun, 2012, 7: 735-738

Pérez-Balado C, Sun H, Griesinger C, de Lera AR, Navarro-Vázquez A. Chem Eur J, 2011, 17: 11983-11986

Intelmann D, Kummerlöwe G, Haseleu G, Desmer N, Schulze K, Fröhlich R, Frank O, Luy B, Hofmann T. Chem Eur J, 2009, 15: 13047-13058

Ge HM, Sun H, Jiang N, Qin YH, Dou H, Yan T, Hou YY, Griesinger C, Tan RX. Chem Eur J, 2012, 18: 5213-5221

Teles RR, França JAA, Navarro-Vázquez A, Hallwass F. Quim Nova, 2015, 38: 1345-1350

Liu LY, Sun H, Griesinger C, Liu JK. Nat Prod Bioprospect, 2016, 6: 41-48

Mevers E, Saurí J, Liu Y, Moser A, Ramadhar TR, Varlan M, Williamson RT, Martin GE, Clardy J. J Am Chem Soc, 2016, 138: 12324-12327

Waratchareeyakul W, Hellemann E, Gil RR, Chantrapromma K, Langat MK, Mulholland DA. J Nat Prod, 2017, 80: 391-402

Fredersdorf M, Kurz M, Bauer A, Ebert MO, Rigling C, Lannes L, Thiele CM. Chem Eur J, 2017, 23: 5729-5735

Liu Y, Saurí J, Mevers E, Peczuh MW, Hiemstra H, Clardy J, Martin GE, Williamson RT. Science, 2017, 356: eaam5349

Cornilescu G, Ramos Alvarenga RF, Wyche TP, Bugni TS, Gil RR, Cornilescu CC, Westler WM, Markley JL, Schwieters CD. ACS Chem Biol, 2017, 12: 2157-2163

Rigling C, Ebert MO. Magn Reson Chem, 2017, 55: 655-661

Yan J, Delaglio F, Kaerner A, Kline AD, Mo H, Shapiro MJ, Smitka TA, Stephenson GA, Zartler ER. J Am Chem Soc, 2004, 126: 5008-5017

Sánchez-Pedregal VM, Santamaría-Fernández R, Navarro-Vázquez A. Org Lett, 2009, 11: 1471-1474

Gayathri C, de la Fuente MC, Luy B, Gil RR, Navarro-Vázquez A. Chem Commun, 2010, 46: 5879

Böttcher B, Schmidts V, Raskatov JA, Thiele CM. Angew Chem Int Ed, 2010, 49: 205-209

Trigo-Mouriño P, Santamaria-Fernández R, Sánchez-Pedregal VM, Navarro-Vázquez A. J Org Chem, 2010, 75: 3101-3104

Swarbrick JD, Ashton TD. Chirality, 2010, 22: 42-49

Kummerlöwe G, Crone B, Kretschmer M, Kirsch SF, Luy B. Angew Chem Int Ed, 2011, 50: 2643-2645

Sun H, d'Auvergne EJ, Reinscheid UM, Dias LC, Andrade CK, Rocha RO, Griesinger C. Chem Eur J, 2011, 17: 1811-1817

Schmidt M, Sun H, Rogne P, Scriba GKE, Griesinger C, Kuhn LT, Reinscheid UM. J Am Chem Soc, 2012, 134: 3080-3083

Trigo-Mouriño P, de la Fuente MC, Gil RR, Sánchez-Pedregal VM, Navarro-Vázquez A. Chem Eur J, 2013, 19: 14989-14997

Vrzal L, Flídrová K, Tobrman T, Dvořáková H, Lhoták P. Chem Commun, 2014, 50: 7590

Xie LG, Bagutski V, Audisio D, Wolf LM, Schmidts V, Hofmann K, Wirtz C, Thiel W, Thiele CM, Maulide N. Chem Sci, 2015, 6: 5734-5739

Fredersdorf M, Göstl R, Kolmer A, Schmidts V, Monecke P, Hecht S, Thiele CM. Chem Eur J, 2015, 21: 14545-14554

Riveira MJ, Trigo-Mouriño P, Troche-Pesqueira E, Martin GE, Navarro-Vázquez A, Mischne MP, Gil RR. J Org Chem, 2015, 80: 7396-7402

Niklas T, Steinmetzger C, Liu W, Zell D, Stalke D, Ackermann L, John M. Eur J Org Chem, 2015, 2015: 6801-6805

Hansmann S, Larem née Montag T, Thiele CM. Eur J Org Chem, 2016, 2016: 1324-1329

Hansmann S, Schmidts V, Thiele CM. Chem Eur J, 2017, 23: 9114-9121

Schwab M, Herold D, Thiele CM. Chem Eur J, 2017, 23: 14576-14584

Naumann C, Kuchel PW. Chem Eur J, 2009, 15: 12189-12191

Marathias VM, Tawa GJ, Goljer I, Bach AC. Chirality, 2007, 19: 741-750

Nath N, Suryaprakash N. Chem Phys Lett, 2010, 496: 175-182

Nath N, Suryaprakash N. J Phys Chem B, 2011, 115: 6868-6875 


\title{
Structural elucidation of organic molecule from residual dipolar couplings
}

\author{
Gaowei $\mathrm{Li}^{2}$, Xinxiang $\mathrm{Lei}^{{ }^{*}}$ \\ ${ }^{1}$ School of Pharmaceutical Sciences, South Central University for Nationalities, Wuhan 430074, China \\ ${ }^{2}$ College of Chemistry and Chemical Engineering, Shangqiu Normal University, Shangqiu 476000, China \\ *Corresponding author (email: xxlei@mail.scuec.edu.cn)
}

\begin{abstract}
Residual dipolar coupling (RDC) has recently become increasingly important in organic structural determination due to their unique information content. This review provides a brief overview of the basic principle and general strategy in the application of RDC in the structure elucidation of organic molecule. The availability of various alignment media and pulse sequences were also discribed. In addition, the applications of RDCs for the constitution, configuration and conformation determination of natural or synthetic molecules were summarized to exemplify the practical application of the technique in recent years. Finally, the challenges and perspectives for future applications were also presented.
\end{abstract}

Keywords: NMR spectroscopy, residual dipolar couplings, alignment media, small organic molecule, structural analysis doi: $10.1360 / \mathrm{N} 032017-00124$ 